\title{
Bubble puzzles: From fundamentals to applications
}

\author{
Detlef Lohse \\ Physics of Fluids Group, Max-Planck Center for Complex Fluid Dynamics, MESA+Research Institute, \\ and J.M. Burgers Centre for Fluid Dynamics, Department of Science \& Technology, University of Twente, \\ P.O. Box 217, 7500 AE Enschede, Netherlands \\ and Max Planck Institute for Dynamics and Self-Organization, Am Fassberg 17, 37077 Göttingen, Germany
}

(Received 19 July 2018; published 21 November 2018)

\begin{abstract}
For centuries, bubbles have fascinated artists, engineers, and scientists alike. In spite of century-long research on them, new and often surprising bubble phenomena, features, and applications keep popping up. In this paper I sketch my personal scientific bubble journey, starting with single-bubble sonoluminescence, continuing with sound emission and scattering of bubbles, cavitation, snapping shrimp, impact events, air entrainment, and surface micro- and nanobubbles, and finally arriving at effective force models for bubbles and dispersed bubbly two-phase flow. In particular, I also cover various applications of bubbles, namely, in ultrasound diagnostics, drug and gene delivery, piezoacoustic inkjet printing, immersion lithography, sonochemistry, electrolysis, catalysis, acoustic marine geophysical survey, and bubble drag reduction for naval vessels, and show how these applications crossed my way. I also try to show that good and interesting fundamental science and relevant applications are not a contradiction, but mutually stimulate each other in both directions.
\end{abstract}

DOI: 10.1103/PhysRevFluids.3.110504

\section{INTRODUCTION}

"How do you find the problems you work on?" "What do you think is the difference between fundamental and applied research?" These are questions I am often asked. My short answer to the first question is, "Be curious!" and to the second one, "In principle, none." Further, what in particular holds for both fundamental and applied problems, both in finding and in solving them?: "Watch, listen, and be open." The answer to both questions can be summarized as follows: "Work on problems you most enjoy. Strange things can happen on the way" (Walter Munk, UCSD). In the best case, the problem to work on is both relevant and outstanding, at the same time.

In this article I want to take the opportunity to give longer answers to these questions, in particular by giving examples from my own scientific biography and scientific journey. As the thread of the article I will choose bubbles. I will report how I first incidentally bumped into the science of bubbles, what and how I learned about them, what wonderful science and great interactions and collaborations with colleagues this endeavor opened for me, and how I kept on bumping into very relevant applications of bubbles in technology. So bubbles have provided me both wonderful scientific problems and very relevant applied questions, to whose solution, I think, we have contributed over the past two and a half decades.

The length scales on which bubbles are relevant range from nanometers to at least tens of meters, and I will give examples for interesting and relevant bubble phenomena on all these scales. The richness of bubble fluid dynamics is reflected in the many dimensionless numbers that are relevant in the context of bubbles [1,2], namely, the

- Reynolds number $\operatorname{Re}=U R / v$, expressing the ratio of inertia forces to viscous forces, where $U$ is the bubble velocity, $R$ its radius, and $v$ the kinematic viscosity. 
- Froude number $\mathrm{Fr}=U / \sqrt{g R}$, the ratio of inertia to buoyancy, where $g$ is the gravitational acceleration.

- Archimedes number $\mathrm{Ar}=g R^{3} \rho\left(\rho-\rho_{g}\right) / \eta^{2}$, the ratio of buoyancy to viscosity, where $\rho$ is the liquid density, $\eta$ its dynamic viscosity, and $\rho_{g}$ the gas density.

- Galileo number $\mathrm{Ga}=g R^{3} / \nu^{2}$, the ratio of gravitational to viscous forces.

- Weber number We $=\rho U^{2} R / \sigma$, the ratio of inertia to capillarity, where $\sigma$ is the surface tension.

- Capillary number $\mathrm{Ca}=\eta U / \sigma$, the ratio of viscous to capillary forces.

- Ohnesorge number $\mathrm{Oh}=\eta / \sqrt{\rho \sigma R}=\mathrm{We}^{1 / 2} / \mathrm{Re}$, the ratio of time of viscous damping to time of the capillary oscillations.

- Eötvös number Eo $=\left(\rho-\rho_{g}\right) g R^{2} / \sigma$, also called the Bond number Bo, ratio of buoyancy to capillarity.

- Stokes number St, the ratio of the characteristic timescale of the bubble to that of the flow.

- Morton number Mo $=g \eta^{4}\left(\rho-\rho_{g}\right) / \rho^{2} \sigma^{3}$, which is a material parameter for a bubble in a certain liquid, depending only on surface tension, density, density contrast, viscosity, and gravity.

- Lewis number Le $=\kappa / D$, the ratio of thermal diffusivity $\kappa$ to mass diffusivity $D$ and thus another material parameter.

- Damköhler number $\mathrm{Da}$, the ratio between the chemical reaction rate and (diffusive or convective) mass transport rate.

- Jakob number $\mathrm{Ja}=\rho c_{p}\left(T-T_{\text {sat }}\right) / \rho_{v} \Lambda$, the ratio of sensible heat to latent heat, where $c_{p}$ is the liquid specific heat, $\Lambda$ is the latent heat, $\rho_{v}$ is the vapor density, $T_{\text {sat }}$ is the saturation temperature of the liquid, and $T$ is the temperature of the surrounding liquid.

-...

We will encounter most of these numbers in this article.

The selection of which bubble problems I will report on is naturally subjective and, as said above, along my own scientific bubble journey. This will also be reflected in the citations, where I will restrict myself to papers that had a significant scientific impact on me and to references to our original work. Some of the given examples I briefly discussed before, in a short proceedings [3] (without Web of Science Index). There are many more bubble problems and applications, which I cannot report here or which I am even not aware of, but I hope that the paper stimulates other scientists to look into the subject and be open to both fundamental and applied bubble problems because, as I hope to be able to show with this article, it is intellectually very rewarding, covers many areas of fluid dynamics, and is extremely relevant in many applications.

\section{SONOLUMINESCENCE: ILLUMINATED BUBBLE DYNAMICS}

The first major scientific bubble problem I bumped into was sonoluminescence. In 1994, during my time as a postdoc at the University of Chicago in the group of Leo Kadanoff, I attended a lecture by Brad Barber on his Ph.D. thesis on single-bubble sonoluminescence [4,5] (later summarized in [6]). This phenomenon had been discovered a few years earlier by Felipe Gaitan [7,8], then a Ph.D. student in Mississippi, when he experimented with an air bubble trapped in a water-filled flask by piezoacoustical forces (the so-called Bjerknes force [9,10]; see Fig. 1 for a similar setup), which at the same time drive the bubble: When the pressure is low, the bubble expands, and once it is high, it is compressed. However, what Gaitan observed was first not believed by anybody: Under certain conditions the bubble can emit light! How can this be? Typical acoustical energies are in the range 


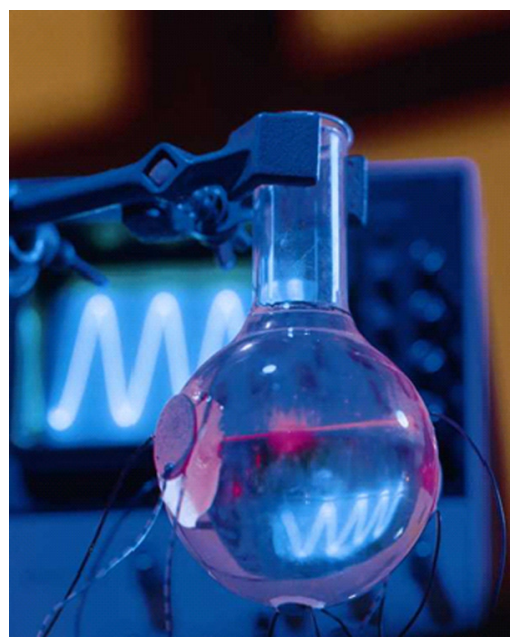

FIG. 1. Setup for single-bubble sonoluminescence. Piezoelectric transducers are glued to a flask filled with water. They excite a standing acoustic wave in which the light-emitting bubble is trapped. The photo was taken by Rüdiger Toegel, Physics of Fluids, Twente, 2000.

of $10^{-12} \mathrm{eV}$ per molecule and typical light energies in the range of $1 \mathrm{eV}$. This means that there is an energy focusing factor of $10^{12}$ ! Sound, radius, and light intensity as a function of time are reproduced in Fig. 2(a).

Directly after Barber's talk, I discussed this fascinating subject with my colleague Michael Brenner, then also a postdoc at the University of Chicago. We asked ourselves the two obvious questions: What is the light-emitting process and under what conditions does this phenomenon happen, i.e., what is the phase space of single-bubble sonoluminescence? We first focused on the second question and started to read and learn about bubble dynamics.

We very soon found the seminal papers by Andrea Prosperetti on this subject, most visibly summarized in his review in Ref. [12]. The core dynamical equation is the celebrated Rayleigh-

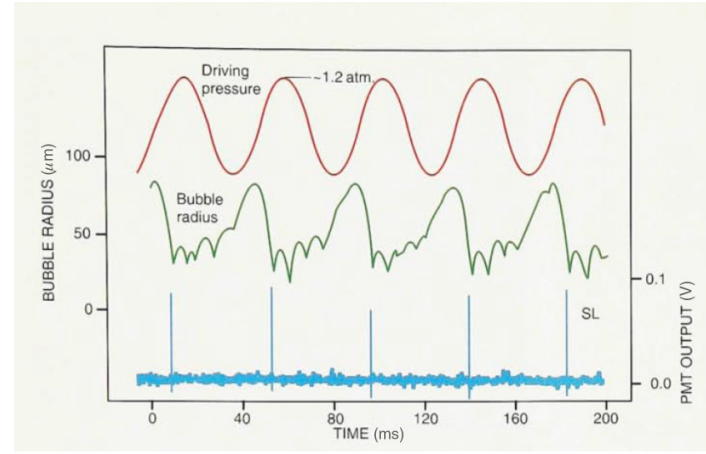

(a)

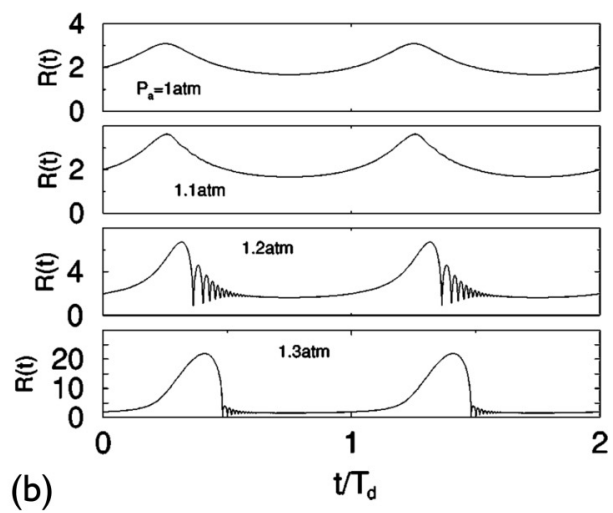

FIG. 2. (a) Acoustic driving pressure $P(t)$ (in red), resulting bubble radius $R(t)$ (in green), and light intensity $I(t)$ (in blue), as measured in Ref. [11]. A negative driving pressure causes the bubble to expand; when the driving pressure changes sign, the bubble collapses, resulting in a short pulse of light (marked SL). The figure is taken from Ref. [8]. (b) Solutions to the Rayleigh-Plesset equation (1) with a sinusoidal driving $P(t)=P_{a} \sin (\omega t)$ at forcing pressures $P_{a}=1.0,1.1,1.2,1.3 \mathrm{~atm}$. The ambient bubble radius is $R_{0}=2 \mu \mathrm{m}$ and the frequency $f=\omega / 2 \pi=26.5 \mathrm{kHz}$. Material parameters are for water at room temperature. 
Plesset (RP) equation for the bubble radius $R(t)$,

$$
R \ddot{R}+\frac{3}{2} \dot{R}^{2}=\frac{1}{\rho}\left(p_{g}-P_{0}-P(t)-4 \eta \frac{\dot{R}}{R}-\frac{2 \sigma}{R}\right),
$$

where $p_{g}(R(t))$ is the pressure inside the gas bubble, $P(t)=P_{a} \sin (\omega t)$ the driving acoustical pressure with amplitude $P_{a}$ and frequency $f=\omega / 2 \pi, P_{0}$ the ambient pressure, $\eta$ the dynamic viscosity, and $\sigma$ the surface tension. A historical review of the development of this equation is given in Ref. [12]. The typical RP bubble dynamics for increasing driving pressure $P_{a}$ is shown in Fig. 2(b).

The left-hand side of the RP equation (1) was already known to Lord Rayleigh, who derived it in the context of an analysis of cavitation damage of ship propellers [1,13,14]. So already in Rayleigh's time applied and fundamental science came hand in hand. The solution to the inertial part $R \ddot{R}+\frac{3}{2} \dot{R}^{2}=0$ of the RP equation (1) is the power law

$$
R(t) \propto\left(t_{s}-t\right)^{2 / 5}
$$

with a diverging singularity in the bubble wall velocity $\dot{R}(t) \propto\left(t_{s}-t\right)^{-3 / 5}$ at time $t_{s}$. This singularity reflects the violent bubble collapse which can occur for strong enough driving (see Fig. 2). In a nutshell, at collapse, the gas inside the bubble gets compressed, heats up, partly ionizes, and at recombination light is emitted $[8,15]$.

The work of Andrea Prosperetti also led us to the conditions under which stable single-bubble sonoluminescence can occur: An obvious necessary condition is the (spherical) shape stability of the bubble, which Eller and Crum [16] had experimentally and Prosperetti theoretically analyzed [17]. We applied his results and determined under what conditions the collapsing bubble would be (spherical) shape stable so that it would on the one hand survive the collapse, but at the same time would still collapse strongly enough so that the gas inside the bubble would be considerably heated [18]. Both a parametric instability and the Rayleigh-Taylor instability turned out to be relevant $[15,18,19]$.

Another necessary condition for stable single-bubble sonoluminescence is the diffusive stability of the bubble. Also the diffusive bubble stability had been analyzed before, namely, in the seminal work by Epstein and Plesset [20], and later extended by Fyrillas and Szeri [21] to oscillating bubbles, for which rectified diffusion [22-24] can occur: For high pressure, the bubble loses gas to the outside liquid, but during the low-pressure period, it can gain gas from outside. For very strong driving the growth can win, mainly due to the thin boundary layer during that time but also due to the much larger bubble size. Applying these ideas to the regime of sonoluminescing bubbles could account for the experimentally observed diffusively stable single-bubble sonoluminescence [25], and combining the conditions of shape stability, diffusive stability, and energy focusing led to the phase diagram of sonoluminescing bubbles [19], which for pure argon bubbles was in good agreement with the experimental observations.

However, air bubbles were found to be stable for 100 times larger gas saturation than pure argon bubbles (see Fig. 3). The reason for this turned out to be the chemical stability of the gas inside the bubble [26]: The bubble is collapsing so strongly that the gas inside is nearly adiabatically compressed. This means that the collapse of the bubble is so violent that no thermal equilibrium with the surrounding water can be established: The bubble is heating up, to about $15000 \mathrm{~K}$, as we now know both theoretically [27] and experimentally [28]. For molecular gases such as $\mathrm{O}_{2}$ or $\mathrm{N}_{2}$ this is much too hot and they dissociate: The resulting radicals react with each other and with the dissociation products of water vapor. $\mathrm{NO}, \mathrm{NH}$, etc., are formed, which dissolve in water. Therefore, it is mainly argon that remains in the bubble, which is contained in air with a concentration of about $1 \%$, explaining the factor of 100 higher gas concentration required for stable single-bubble sonoluminescence (SBSL) with air as compared to the pure argon (or any other inert gas) case. This theory later got confirmed through various experimental results (see, e.g., [29,30]). Another advantage of argon for achieving strong SBSL is that, in contrast to $\mathrm{O}_{2}$ or $\mathrm{N}_{2}$, argon has no internal 

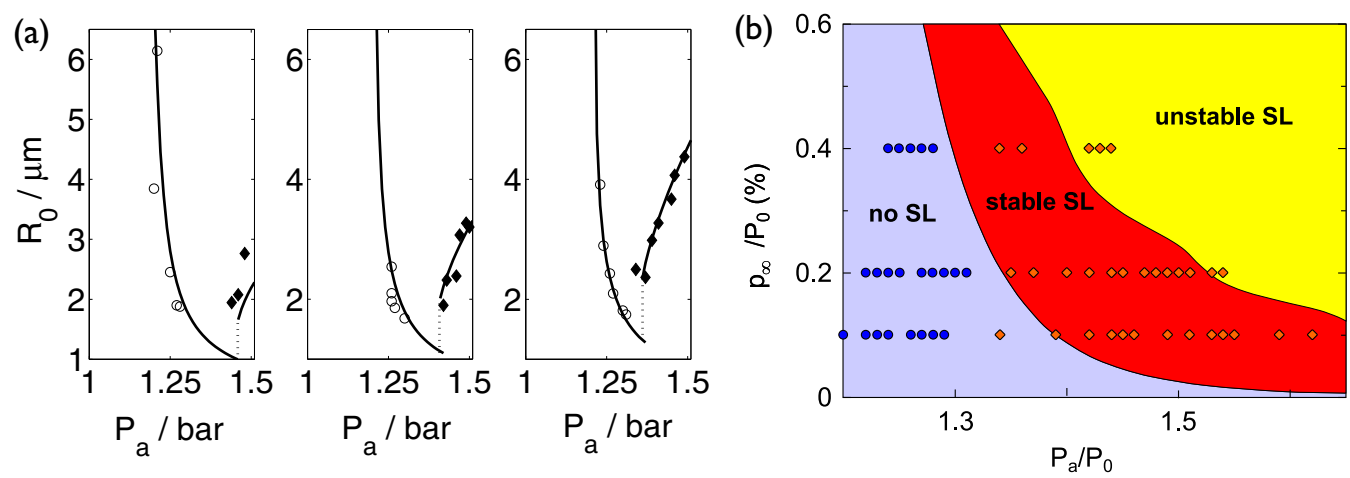

FIG. 3. (a) Phase space of single-bubble sonoluminescence in the argon concentration $P_{\infty} / P_{0}$ vs driving pressure $P_{a} / P_{0}$ phase plane for pure argon bubbles, showing the three phases, no SL, stable SL (only possible for very small argon concentrations), and unstable SL, where the bubbles grow by rectified diffusion while emitting light and finally run into a shape instability [19]. Adopted from [19]. (b) Phase space of single-bubble sonoluminescence in the bubble radius vs driving pressure phase plane for air bubbles, taken from Ref. [27]. The bubble equilibrium radius $R_{0}$ versus driving pressure $P_{a}$ is shown for three different air concentrations $(10 \%, 20 \%$, and $40 \%$ of saturation; the driving frequency is $33.4 \mathrm{kHz})$. The curves, signaling stable bubbles, follow from our parameter-free theory [27]; the data points had been measured by Ketterling and Apfel [30]. The bubble emits light only on the right curves where argon has accumulated in the bubble (closed diamonds). Shown on the left curves are the losses by chemical reactions and the growth by rectified diffusion balance.

degrees of freedom. Thus the focused energy of the bubble collapse can directly be transferred into heat. The $15000 \mathrm{~K}$ which is reached in this way is sufficient to partly ionize the gas. Recombination of ions and electrons leads to light emission through thermal bremsstrahlung [31,32]. Later we extended the ideas of chemical stability of Ref. [26] to include various other chemical reactions of air with water [27] to find very good quantitative agreement between experimental and theoretical phase diagrams [see Fig. 3(b)].

In a sense, single-bubble sonoluminescence can be viewed as illuminated bubble dynamics, with the RP dynamics as backbone. A combination of concepts from hydrodynamics (both shape stability and diffusive stability), chemistry, plasma physics, applied mathematics, thermodynamics, and acoustics led to the phase diagrams, which are in good agreement with the experiments $[15,27]$. In its conceptual simplicity, an isolated, fixed, noninteracting single bubble in a flask, it can also be seen as a hydrogen atom of bubble fluid dynamics, on which we learned a lot.

Our work on sonoluminescence started off as pure fundamental research, driven by curiosity. We had not asked ourselves whether there would be any applications. Single-bubble sonoluminescence simply was a fascinating and outstanding problem, with major open questions. In answering them, we learned tremendously, including on

- Acoustic and other forces on bubbles

- Bubble dynamics and bubble shape stability

- Diffusive bubble stability

- Chemical bubble stability

- Bubble nucleation

- Bubble collapse and cavitation

- Plasma formation and thermal bremsstrahlung 
As we will see, all these items turned out to be very relevant in connection with various applications, sometimes very unexpectedly. The insight obtained from single-bubble sonoluminescence therefore helped us enormously to discover and identify applied problems and to help to solve them or at least make some progress on them. In the following sections I will report how this came about.

Namely, I will report on:

- How our understanding of the bubble dynamics and of the bubble shape stability [subject (2) in above list] contributed to ultrasound diagnostics and to improve ultrasound contrast agents, as well as other applications of bubble dynamics in the medical context (Sec. III).

- How we found out that the sound of snapping shrimp originates from a cavitating bubble [Sec. IV, originating from subjects (5) and (6) in the above list].

- How the collapse of a bubble or a void close to a surface focuses the energy, leading to a major jet [Sec. V, originating from subject (6) in the above list].

- How an entrained bubble in a piezoacoustic inkjet channel can cause major trouble due to rectified diffusion [subject (3) in the above list] and how to solve this problem (Sec. VI).

- How bubbles can nucleate on a microstructured surface and, when acoustically driven, collapse in a controlled way, enhancing the efficiency of ultrasonic cleaning and chemical reactions [Sec. VII, originating from subjects (4)-(6) in the above list].

- How our understanding of diffusive bubble stability [subject (3) in the above list] was instrumental in figuring out why surface nanobubbles and surface nanodroplets are stable, with various applications in electrolysis, catalysis, diagnostics, and the food and remediation industry (Sec. VIII).

- Finally, how our understanding on bubble forces [subject (1) in above list] brought us to bubbly two-phase flow, including studying drag reduction in turbulent bubbly flow, for which bubble deformability [subject (2)] is crucial (Sec. IX).

The paper closes with conclusions and with a short outlook (Sec. X). In particular, I will put forth motivation why, from my point of view, we live in the golden age of fluid dynamics. Both wonderful bubble science and very relevant bubble applications are ahead of us.

\section{ULTRASOUND DIAGNOSTICS, ULTRASOUND CONTRAST AGENTS, AND OTHER APPLICATIONS OF BUBBLES IN DIAGNOSTICS, THERAPY, AND MEDICINE}

In an outreach effort, in order to popularize physics, fluid dynamics, and bubbles, in 1995 I had written an article on single-bubble sonoluminescence in Physikalische Blätter, which was the German analog of Physics Today. Based on this, I got contacted by a physicist working for a pharmaceutical company on ultrasound contrast agents (UCAs), which are introduced into the blood to enhance the acoustic scattering and which contain small encapsulated microbubbles. These very effectively scatter ultrasound [see Fig. 4(a)]. In this way, it is, e.g., possible to visualize the perfusion of tissue, like the heart muscle. The images are meanwhile used to obtain diagnostic information from the volume, shape, and movement of the heart ventricles, in studying the blood flow in small blood vessels, in blood perfusion measurements, and in targeted molecular imaging, among others [33-35].

One of the nagging questions in ultrasound diagnostics in the mid 1990s was, how does one increase the signal-to-noise ratio? Namely, when detecting the emitted sound from the bubble at the driving frequency, the signal is obscured by the reflections from tissue. To improve the signal quality, it had been proposed [40] to detect higher harmonics of the driving frequency in the sound emission spectrum of the bubble. Conversely, also subharmonics had been suggested for better contrast. The immediate question was, what bubble properties are optimal for these purposes, in particular what bubble size, for given driving frequency? This indeed was the question with which the pharmaceutical industry approached us, and thanks to our work on single-bubble 
(a)
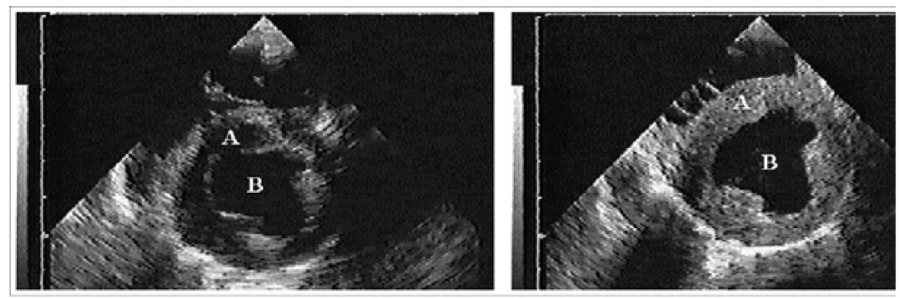

(b)

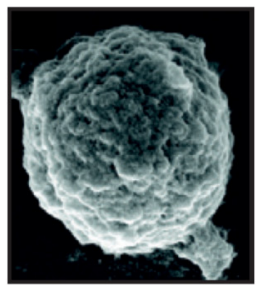

$\sim 0.5 \mu \mathrm{m}$
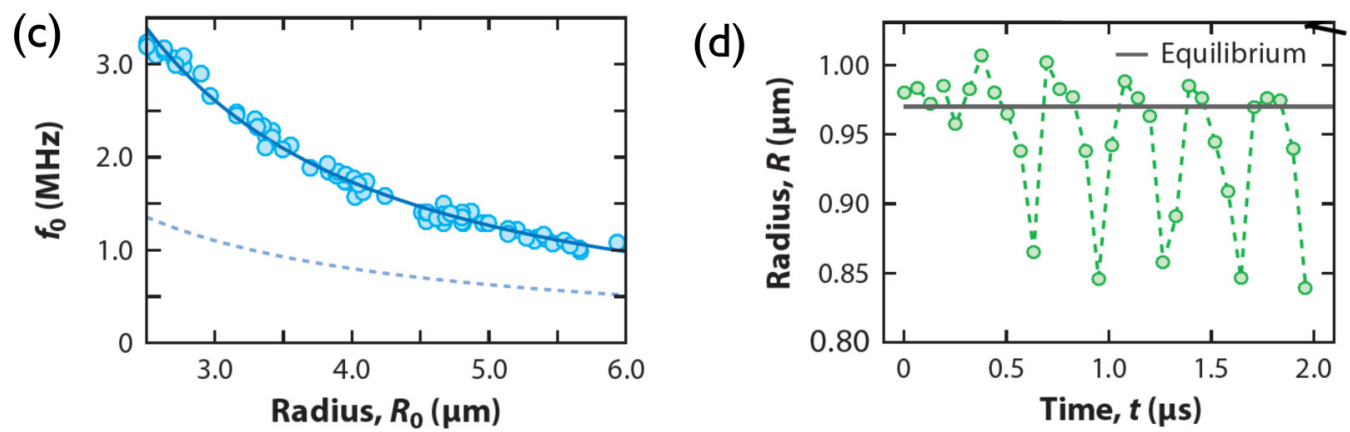

FIG. 4. (a) Ultrasound image of a heart without (left) and with (right) previous injection of microbubbles. In the second case the structures become clearer as the bubbles act as ultrasound contrast enhancers. (b) Electron micrograph of a microbubble coated with a protein layer, taken from Ref. [36]. (c) Eigenfrequency $f_{0}$ of a sound-driven coated with DPPC monolayer as a function of the ambient bubble radius (blue data points, from [37]). The solid blue line shows the fit to the Marmottant model [38], whereas the dashed line shows the Minnaert frequency (3). The figure has been adopted from Ref. [39]. (d) With the coating the sound-driven bubbles show the typical compression-only behavior, with the compression being much more pronounced than the expansion. The figure has been taken from Ref. [38].

sonoluminescence, we could straightforwardly provide an answer, namely, by simply solving the RP equation with the relevant parameter for medical ultrasound imaging: Here, rather than in the range of $20 \mathrm{kHz}$ to $30 \mathrm{kHz}$ as common for single-bubble sonoluminescence, the typical driving frequencies are between 2 and $10 \mathrm{MHz}$ and typical bubble radii are a few micrometers. From linearizing the RP equation (1) it follows that the eigenfrequency of the volume oscillations of an acoustically driven bubble with ambient radius $R_{0}$ and under isothermal conditions approximately is $[1,12,41]$

$$
\omega_{0}=\sqrt{\frac{3 P_{0}}{\rho_{l} R_{0}^{2}}} .
$$

This eigenfrequency is called Minnaert frequency. With the material parameters for water under ambient conditions this gives the well-known rule of thumb [41]

$$
f_{0} R_{0} \approx 3 \mathrm{MHz} \mu \mathrm{m}=3 \mathrm{kHz} \mathrm{mm}=3 \mathrm{~Hz} \mathrm{~m}
$$

for the resonance frequency $f_{0}=\omega_{0} / 2 \pi$. For the frequencies of medical ultrasound imaging, the resonance radii are thus in the micrometer range. Also in the fully nonlinear case, thanks to the full RP equation, we could calculate optimal parameter values for maximal sound emission in the second harmonic and in subharmonics and could make statements about the expected bubble shape stability in those regimes [42].

One issue we had first ignored was that the ultrasound contrast agent bubbles are not "naked", but are coated with lipids and polymers [Fig. 4(b)], to avoid the obviously undesirable bubble clustering in the body and to increase their lifetime. The coating, however, modifies the oscillation behavior of the bubble in an a priori unknown way. Therefore, a few years later, to take the effect of the 


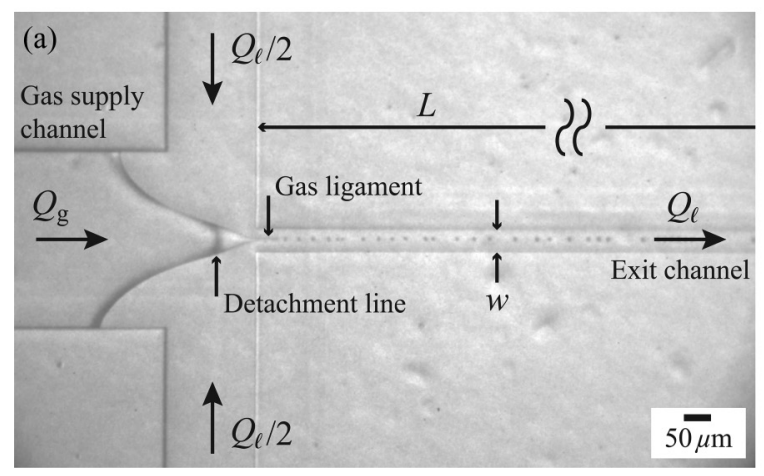

(b)

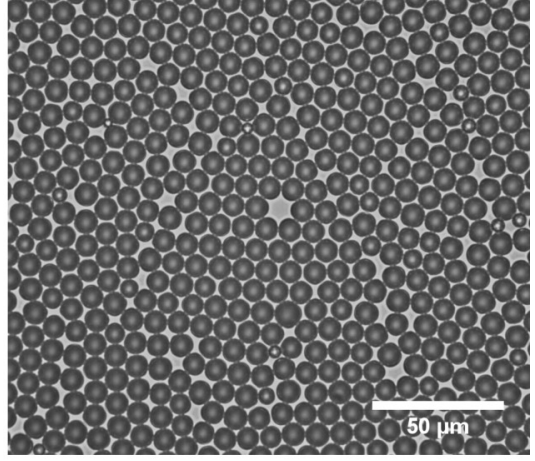

FIG. 5. (a) Principles of the coflow device of Ref. [54] to produce monodisperse microbubbles. The control parameters are the gas flow rate $Q_{g}$, the liquid flow rate $Q_{\ell}$, and the geometric parameters, including the thickness (not shown) of this quasi-2D device. The scale bar on the bottom right is $50 \mu \mathrm{m}$. The figure has been taken from Ref. [54]. (b) Monodispersed ultrasound contrast agents produced with such a coflow device, employing the principles of Ref. [54]. The scale bar in the bottom right corner is $50 \mu \mathrm{m}$. The figure is by Wim van Hoeve, Tide Microfluidics, Enschede.

coating on the bubble dynamics into consideration, we developed a model to quantitatively describe this modification of the RP dynamics (1), which is now known as the Marmottant model [38]. The key idea behind this model is to introduce an instantaneous bubble-size-dependent surface tension, reflecting the buckling behavior of the bubble coating when compressing the bubble, an elastic regime, and a shell-ruptured regime. An excellent recent review of such modified bubble dynamics can be found in Ref. [39].

To experimentally test such models for the relevant frequencies in the megahertz regime, one unavoidably needs ultrahigh-speed imaging, with frame rates much greater than $1 \mathrm{MHz}$. In an effort led by Nico de Jong and Michel Versluis, we therefore developed $[43,44]$ an ultrafast camera, which allows imaging 128 digital frames with a frame rate of up to $25 \mathrm{MHz}$. We called it Brandaris 128 , as it is based on a rotating mirror, just as the famous Dutch lighthouse Brandaris on Terschelling. This camera allowed us to gain insight into the volume and shape oscillations of ultrasound contrast agent bubbles [45-47], in particular when combining them with measurements of the acoustic emission of such coated bubbles [45]. This procedure allowed us to adjust the model parameters of the Marmottant model [38] to the experimental data, very nicely reflecting the observed so-called compression-only behavior of the UCA bubbles [38,45,48,49] [see Figs. 4(c) and 4(d)], which is nothing else than (thanks to the bubble coating) modified RP dynamics, thus giving the ultrasound contrast agent community a very relevant tool.

Another necessity which arose out of the applications of bubbles as ultrasound contrast agents was to produce large numbers of relatively monodisperse and coated microbubbles. The monodispersity is desirable to enhance the scattering property of the bubbles, which is optimal close to the bubble resonance size given by Eq. (4). We achieved this with a so-called coflow device originally developed by Howard Stone, Dave Weitz, and co-workers [50-52] and in the case of bubbles by Gordillo et al. [53], but now operated in a regime in which we could produce particularly small monodisperse bubbles in large quantities [54] (see Fig. 5). Meanwhile this method has been commercialized within a start-up company, as spin-off from our Physics of Fluids group.

The development of the Brandaris 128 ultrahigh-speed imaging facility also allowed us to study the interaction of ultrasonically driven bubbles with cells. This interaction is sometimes spectacular, as can be seen in Fig. 6 [55], which shows a HeLa cell culture (a commonly used human cell line) grown on a glass plate, just after a bubble has collapsed close to it. The collapsing bubble exerts such strong shear forces on the cell that they detach from the glass plate, or, if they are more remote, holes in the cell membrane are induced. These holes, which can close again after some 


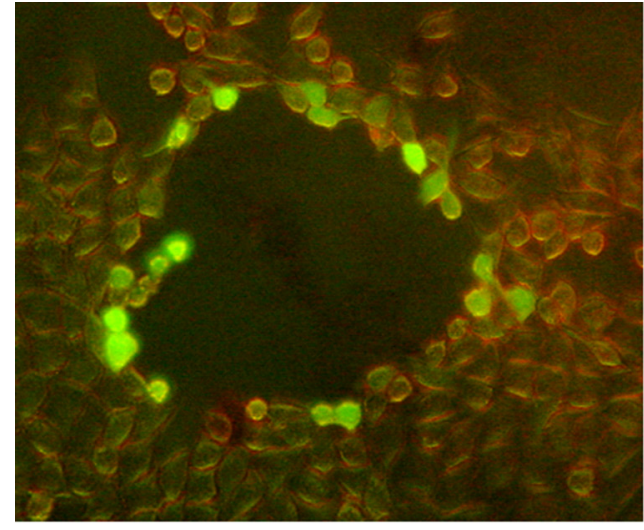

(a)

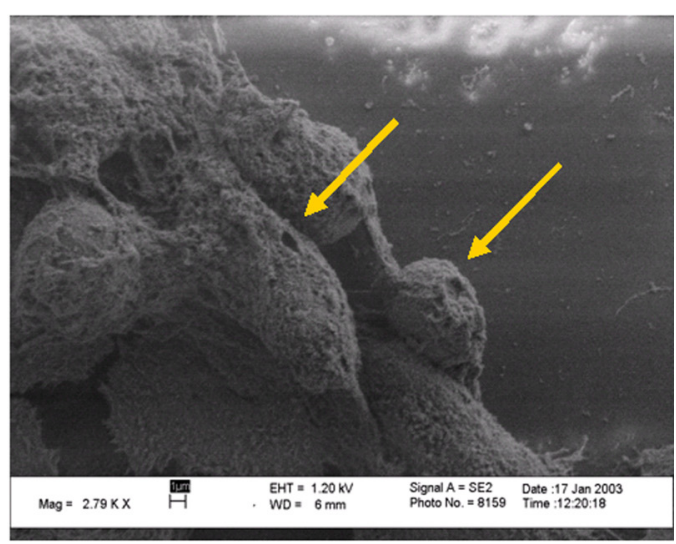

(b)

FIG. 6. (a) HeLa cells (several micrometers in size) glued to a glass plate. In the center of the cell colony a bubble imploded, leading to a cell detachment due to the induced shear flow. Cells at the edge took up fluorescein, which is only possible through holes in the cell membrane. (b) These holes can be visualized through electron microscopy. The figures have been taken from Ref. [55].

time, allow drugs or genes to invade the cell. Therefore, ultrasonically driven bubbles can be used for local application of genes or drugs. This includes employing emulsions of droplets composed of liquid perfluorocarbons, which are acoustically activated to undergo a phase change into a bubbly dispersion, a procedure termed acoustic droplet vaporization [56].

I take the opportunity to stress the fundamental differences between vapor and gas bubbles, which in detail are elaborated and explained by Prosperetti in his recent review on vapor bubbles [57]. While for gas bubbles it makes sense to ascribe them an ambient radius as the gas exchange processes with the environment are slow due to the slow gas diffusion, it does not make sense for vapor bubbles, which are controlled by the much faster heat diffusion and condensation and evaporation. Also the resonance frequency of vapor bubbles does not scale like the inverse radius as for gas bubbles [Eq. (4)], but as $\sim 1 / R^{2 / 3}$ [57]. Note that an expanding vapor bubble is invaded not only by evaporating liquid, but also by gas dissolved in the liquid, which in the long term crucially determines its dynamics and lifetime, as we showed for vapor bubbles generated with water-immersed plasmonic nanoparticles [58].

Such so-called plasmonic microbubbles $[59,60]$ indeed also have potential biomedical applications [61-65], again in both diagnosis and therapy (next to other potential applications in micro- and nanomanipulation, catalysis, and solar energy harvesting [66]), and understanding and controlling the dynamics of these microbubbles is key to successfully exploit them, and to recognize potential risks. In Fig. 7(a) we show the life cycle of such a plasmonic nanobubble, nucleating in air-saturated water thanks to laser illumination of plasmonic gold nanoparticles, each with a diameter of about $100 \mathrm{~nm}$. Note the very different timescales in between the four snapshots. After some delay time $\tau_{d}$ after the beginning of the illumination, the bubble explosively grows to giant size (as compared to the size of the nanoparticle), up to a maximum radius of $80 \mu \mathrm{m}$, and collapses again within approximately $10 \mu \mathrm{s}$ [bubble life phase 1 , which we time resolved with ultrahigh-speed imaging in Fig. 7(b)]. The maximum bubble volume $V_{\max }$ remarkably increases with decreasing laser power $P_{\ell}$ [see Fig. 7(c)] and, also remarkably, decreases with increasing gas saturation of the water.

We could explain [67] these remarkable features, based on the phase diagram of water [see Fig. 7(d) for a sketch] and in particular the lines of attainable superheat therein, which are in between the line of liquid-vapor coexistence and the liquid spinodal line. We first measured the delay time $\tau_{d}$ from the beginning of the illumination up to nucleation, which drastically increases with decreasing laser power, leading to less total dumped energy $E=P_{\ell} \tau_{d}$. This dumped energy 


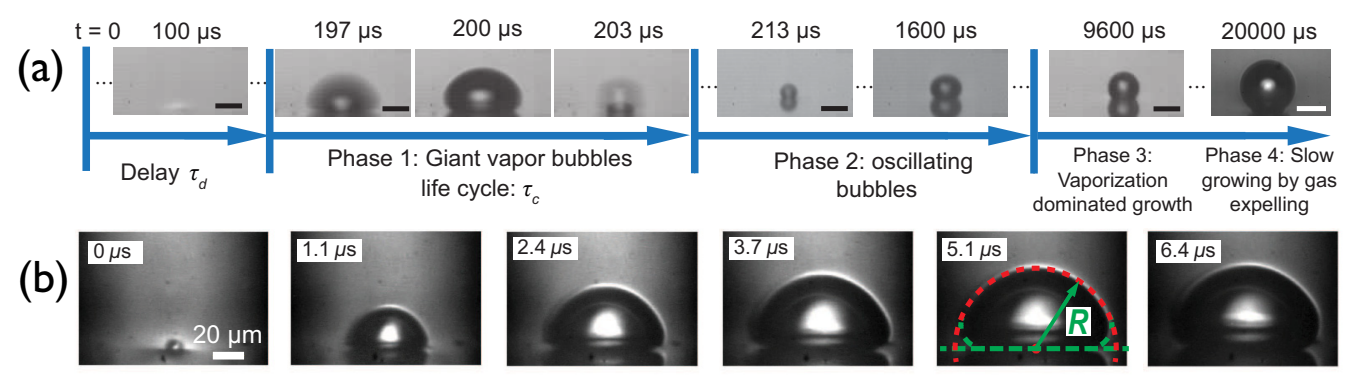

(c)

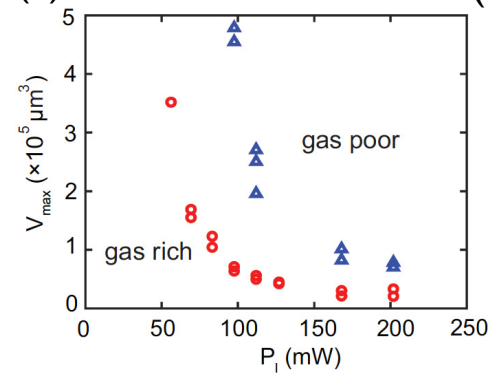

(d)

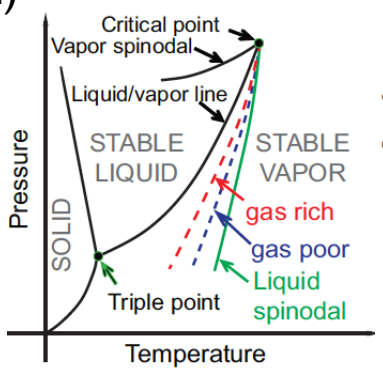

(e)

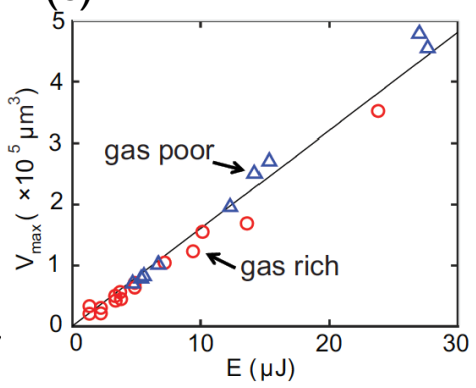

FIG. 7. (a) Time sequence of a plasmonic bubble (generated at gold nanoparticles) under continuous laser irradiation in gas-rich water. The nucleation and growth dynamics of the plasmonic bubbles are shown as four phases (see the text). The scale bar is $25 \mu \mathrm{m}$. (b) Evolution of the initial giant plasmonic bubble during its life cycles in an air-saturated liquid, captured at $7.47 \times 10^{6}$ frames $/ \mathrm{s}$. The laser power was $P_{\ell}=185 \mathrm{~mW}$. (c) Maximum volume $V_{\max }$ of the giant bubble as a function of laser power $P_{\ell}$ in gas-rich water and gas-poor water. (d) Schematic phase diagram of water. The green solid line is the liquid spinodal line, the theoretical limit of superheat, while the blue and red dashed lines schematically depict the attainable superheat for gas-poor and gas-rich water, respectively. (e) Maximal volume of the giant bubble $V_{\max }$ as a function of the dumped energy $E=P_{\ell} \tau_{d}$ in gas-rich and gas-poor water. Both cases show the identical linear relation, regardless of the delay time $\tau_{d}$ and the applied laser power $P_{\ell}$. All figures have been taken from Ref. [67].

$E$ shows a universal linear scaling relation with $V_{\max }$, irrespectively of the gas concentration of the surrounding water [Fig. 7(e)]. This finding supports the interpretation that the initial giant bubble is a pure vapor bubble. In contrast, the delay time does depend on the gas concentration of the water, as gas pockets in the water facilitate an earlier vapor bubble nucleation, which leads to smaller delay times and lower bubble nucleation temperatures [see again the phase diagram of water, Fig. 7(d)]. After the collapse of the initial giant bubbles, first much smaller oscillating bubbles form out of the remaining gas nuclei [bubble life phase 2, up to typically $10 \mathrm{~ms}$; see Fig. 7(a)]. Subsequently, a vaporization-dominated growth phase takes over and the bubble stabilizes (life phase 3 ). In the final life phase 4 , the bubble slowly grows by gas being expelled due to heating of the surrounding.

\section{SNAPPING SHRIMP AND THE UNDERWATER SOUND OF BUBBLES}

In another outreach effort, in 1999 I gave a colloquium talk on single-bubble sonoluminescence at the Technical University of Munich (TUM), also addressing the issue of sound emission from the collapsing bubbles. After the talk, I met with a TUM zoologist who showed me a signal of the sound emission of so-called snapping shrimp, and the same evening I met these animals in the laboratory. They are about $5 \mathrm{~cm}$ long and live in the tropical ocean. With the help of a huge claw they can make considerable noise. This animal is very unpopular with the navy: First, it disturbs underwater communication between submarines. Second, even worse, hostile submarines use shrimp colonies to "acoustically hide" themselves. 

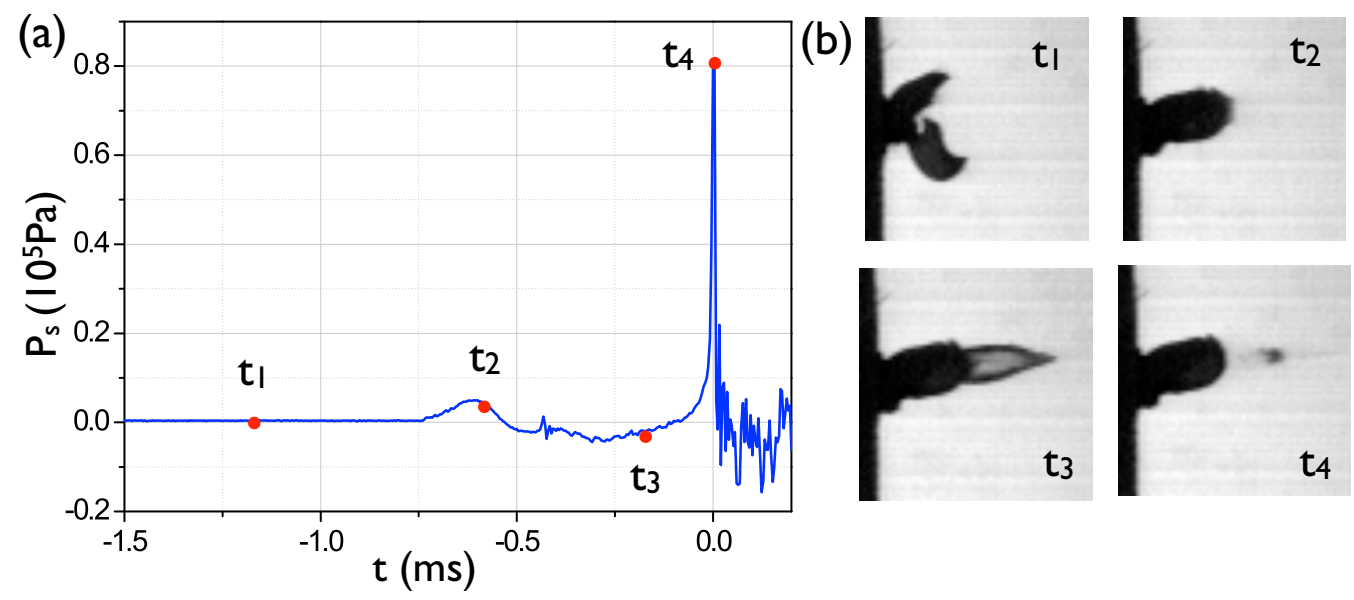

FIG. 8. (a) Sound emission of a snapping shrimp as a function of time. (b) The frames show the closing claw at the four times $t_{1}, t_{2}, t_{3}$, and $t_{4}$ indicated in (a). At $t_{2}$ the claw is closed, but there is no sound. At $t_{3}$ the cavitation bubble has nucleated and grown. The bubble collapse at $t_{4}$ coincides with the maximum of the sound emission. The figure has been adopted from Ref. [69].

The first obvious question to ask is, how does the shrimp make such noise? Zoologists thought that the sound pulse is caused by mechanical vibration on claw closure. Knowing the sound emission of a collapsing bubble from the work on sonoluminescence, I had my doubts on this hypothesis. Moreover, I knew the beautiful paper of Prosperetti, Crum, and Pumphrey on the underwater noise of rain [68], in which it is shown, by correlating high-speed imaging and sound detection with a hydrophone, that the noise arising when raindrops fall on a water surface does not originate from the impact, but from the oscillations of an entrained bubble. Following their example, we made high-speed movies of the snapping event (the shrimp had to be tickled) and correlated them with the corresponding sound track [69]. What we saw was that the shrimp closes its claw so quickly that a fast water jet develops. High velocities imply low pressure. Just as in single-bubble sonoluminescence, this leads to growth of bubbles. Once the pressure has equilibrated, the bubble collapses, leading to sound emission (Fig. 8) at bubble collapse,

$$
P_{s}(r, t)=\frac{\rho R}{r}\left(2 \dot{R}^{2}+R \ddot{R}\right) .
$$

Indeed, the singularity (2) in the RP bubble dynamics is reflected in a singularity $P_{s}(t) \sim\left(t_{s}-\right.$ $t)^{-6 / 5}$ in the sound emission.

The second obvious question to ask is, why is the snapping shrimp doing all this? The answer is simple: It wants to eat! The emitted sound pulse of the collapsing bubble is so strong that little fish or shrimp get stunned or even killed by it and are then eaten up by the snapping shrimp. Our explanation immediately solved another paradox: Why are there no snapping shrimp in the deep

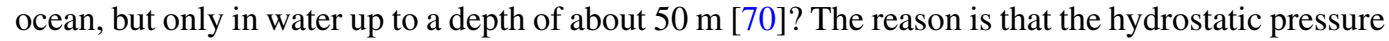
increases with increasing depth so that eventually the shrimp can no longer generate a cavitating bubble. Thus it would starve in deeper water.

With our background in single-bubble sonoluminescence, we could not resist looking into possible light emission from the shrimp-produced collapsing bubbles: Indeed, there was a faint light emission, a phenomenon we called shrimpoluminescence [71].

Sound emission from oscillating or collapsing bubbles is relevant not only for snapping shrimp, but also on a much larger length scale. Equation (4) not only is valid in the micrometer or millimeter range, but can even be employed as an estimate in the meter range, implying that the resonance frequency of a bubble with an ambient radius $R_{0}=1 \mathrm{~m}$ is $3 \mathrm{~Hz}$. Such extremely low frequencies 


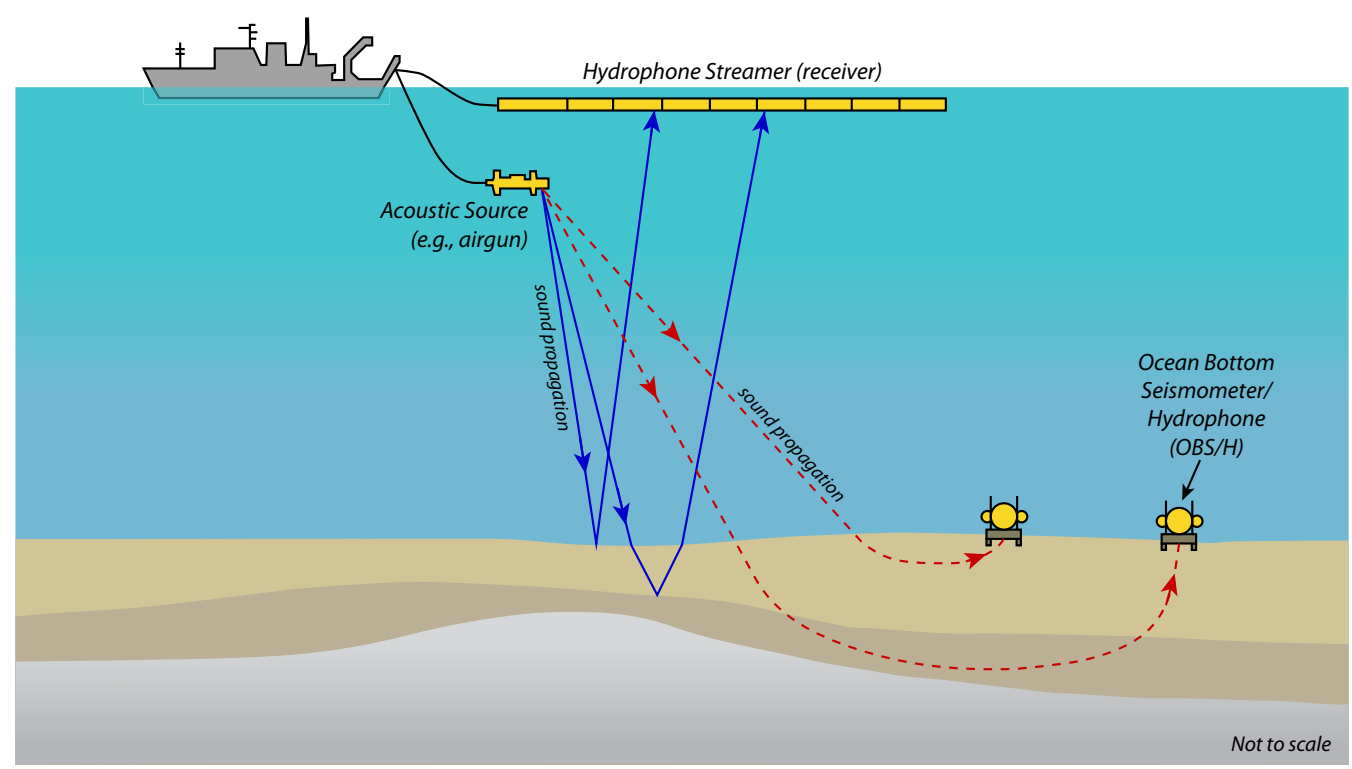

FIG. 9. Principle of acoustic marine geophysical survey. Illustration is a courtesy of National Science Foundation.

are of great interest, for example, in the oil industry for acoustic marine geophysical exploration. The giant bubbles are generated with so-called air-gun acoustic sources. Their operating principle is as follows: During the charging process, air is put under very high pressure in a cavity in the air gun. This air is laterally released during the discharging process, leading to a giant air bubble with typical diameters of one meter and beyond. The oscillations of this air bubble lead to sound generation and emission, according to Eq. (5). The sound gets reflected at the ocean bottom and is detected both with hydrophone streamers and with ocean-bottom seismometers and hydrophones (see Fig. 9). To extract the relevant information from these data, low frequencies around $1 \mathrm{~Hz}$ are crucial, whereas high frequencies beyond $120 \mathrm{~Hz}$ are attenuated in the earth. This is the reason why it is essential to generate low frequencies with the air gun (or air-gun clusters) and thus employ big bubbles.

Bubbles of this size will no longer be strictly spherical during their whole period of life, and this will affect their sound emission behavior with respect to both intensity and direction. The way to calculate the dynamics of such large bubbles or voids from the Navier-Stokes equation (or, to be precise, from its potential flow approximation) is boundary integral methods, in the context of fluid dynamics, again pioneered by Andrea Prosperetti [72]. With such methods one can optimize the sound emission in the low-frequency range. This is not restricted to single bubbles, but one can also analyze bubble clusters and their collective sound emission, either in two dimensions by employing some symmetry or in fully three-dimensional (3D) systems. Presently, together with the oil company Shell, who contacted us on this problem, we are pursuing such calculations.

\section{IMPACT ON LIQUIDS AND ON SOFT SAND}

We had started to work with boundary integral methods in the context of impact events. What had triggered us was again the above-mentioned pioneering paper by Prosperetti, Crum, and co-workers on rain drops falling on the ocean [68,72], leading to bubble entrainment and sound emission. Apart from the question on sound emission, another important question to ask is, how much air ends up in the water? The answer is relevant, for example, in climate models, in which models for the atmosphere are coupled to those of the ocean. To answer this question, Prosperetti and 
(a)

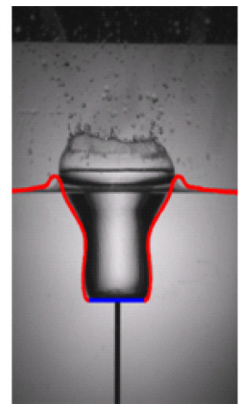

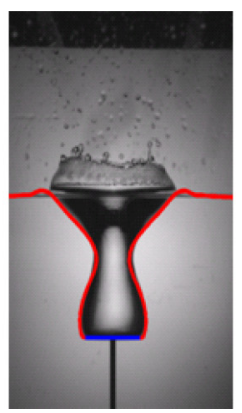
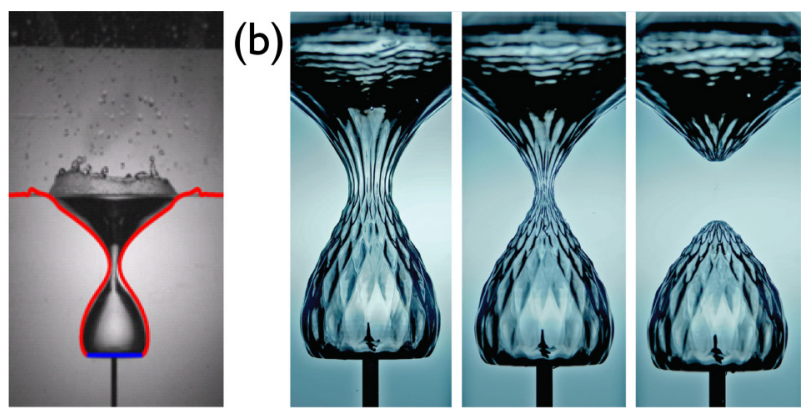

FIG. 10. (a) A disk (diameter $6 \mathrm{~cm}$ ) is pulled through an air-water interface with a constant velocity of $1 \mathrm{~m} / \mathrm{s}$. The emerging void is collapsing due to the hydrostatic pressure. The photos were taken 88,115 , and $131 \mathrm{~ms}$ after the impact of the disk; the solid line results from a boundary integral calculation without any free parameter and shows excellent agreement with the data. The pictures have been taken from Ref. [79]. (b) Here the pulled impact disk (diameter $4 \mathrm{~cm}$ ) has a small azimuthal asymmetry with a mode $m=20$ and an amplitude of $4 \%$. Again, the impact velocity is $1 \mathrm{~m} / \mathrm{s}$. The three snapshots show the evolution of the shape distortions. The photo has been taken from Ref. [86].

co-workers had employed boundary integral methods [72-74], finding very good agreement with the experimental results.

In the late 1990s and in the first two decades of this century, the development of digital high-speed cameras has boomed, ever increasing in frame rate, resolution, and storage and lowering in price considerably. That gave us the opportunity to look into the impact events and the subsequent void collapse in more detail. This line of research was also triggered by single-bubble sonoluminescence, namely, to analyze in detail the hydrodynamic singularity at collapse; here not the spherically symmetry bubble collapse, but the axially symmetric void collapse. In fact, mathematically, the collapse of the void formed after impact can approximately be described by a two-dimensional Rayleigh equation [75-79] analogous to the 3D Rayleigh equation (1), which has been so successful in describing the collapse of the sonoluminescing bubble. The $2 \mathrm{D}$ version of the inertial part of the Rayleigh equation reads $R \ddot{R}+\dot{R}^{2}=0$, with the singularity solution $R(t) \propto\left(t_{s}-t\right)^{1 / 2}$. However, the collapse of a void emerging at impact is not purely two dimensional and correction terms emerge. Indeed, experimental studies have found that the exponent of the power law is higher than $1 / 2$ (typical values found are 0.54-0.60) [77-82] and theoretical studies have shown that the exponent indeed has a weak dependence on the logarithm of the remaining collapse time, approximating to $1 / 2$ only asymptotically at the end [83-85].

To analyze these questions in a controlled way, rather than letting droplets or spheres fall on a water surface, we pulled a disk with controlled velocity $V$ (defining the Froude number $\mathrm{Fr}=$ $V^{2} / g R_{\text {disk }}$ as a dimensionless control parameter) through the air-water interface and performed high-speed imaging of the void collapse and jet formation $[79,82,85]$. Figure 10(a) shows how the cavity develops and then collapses due to the hydrostatic pressure from the side. At singularity two jets emerge (Fig. 11): one upward, straight into the air, the other downward into the developing bubble. Just as in three dimensions, the focusing power of the collapsing (sonoluminescing) bubble is converted into sound and light emission (and of course heat); in two dimensions this focusing power is converted into the jet formation. As one can see from Fig. 10(a), excellent agreement between experiments and the boundary integral simulations can be achieved. Even the effect of the air flow can be included [87], which reverses during the collapse from downward during void formation to upward during void collapse, leading to a supersonic air flow out of the closing void.

The analogy between the 3D bubble collapse and the 2D void collapse goes so far that even the shape stability can be analyzed in one-to-one analogy, as again first done by Prosperetti [88]. Later, we extended this analysis both theoretically and experimentally to find the shape of a collapsing nonaxisymmetric impact-created air cavity in water [86] [see Fig. 10(b)]. 


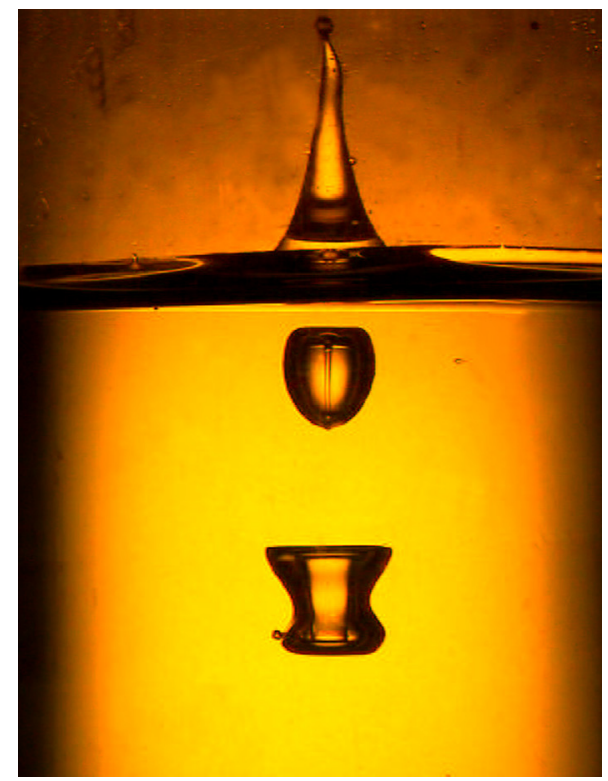

FIG. 11. Comparable process to Fig. 10 at a later stage. Two jets have developed at the singularity: one upward and one downward into the bubble. This photo has been taken from Ref. [14].

To proceed to even larger scale, how comparable is the impact of a ball on a water surface to that of an asteroid on the surface of a planet? We downscaled such an astroid impact to laboratory scale by fluidizing very fine sand. Before the impact event, the airflow is turned off. The first reason for the fluidization is to create reproducible conditions. Second, the fluidization implies that the energy stored in the ground decreases by orders of magnitude, corresponding to the much smaller kinetic energy of the falling ball in the laboratory as compared to that of an impacting astroid. The idea is to achieve similar Froude numbers and Newton numbers (the ratio between yield stress of the surface and kinetic energy of the intruder) as in the geophysical event, hoping for similar dynamical behavior.

Indeed, the phenomena of the impact of a ball on such prepared sand turned out to be very comparable to those of the impact on water [76]: First a splash is formed and then a jet develops (Fig. 12), just as in the water case (Fig. 11). Even the bubble, which forms in water, again develops and slowly rises, finally, when hitting the sand-air interface, causing a granular eruption. It however also turned out that the air in between the sand grains has a major role in the emergence and intensity of the jet [89-91], namely, when prior to the impact event (partially) evacuating the air from the container with the sand, the jet is much less pronounced as the impacting object can intrude less deep, leading to a weaker "hydrostatic" collapse. An excellent review on impact of objects on granular beds can be found in Ref. [92].

\section{PIEZOACOUSTIC INKJET PRINTING AND IMMERSION LITHOGRAPHY}

Science in the university and science in industry often have a difficult time finding each other. In an effort to facilitate the contact, in 2001 the Dutch Science Foundation organized a get-into-contact event, where I met Hans Reinten from Océ, at the time an independent Dutch company, and since 2010 a member of the Canon Group. Today, Océ is the leading developer of high-end production printing systems for commercial printing. Océ has been developing piezoacoustic inkjet printers and obviously various great fluid dynamics challenges come with this, starting from the flow in the nozzle, to the jetting and droplet process, down to drop impact, drop spreading, and drop-paper 


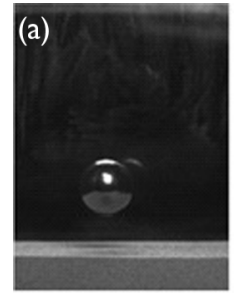

$t=-7 m s$

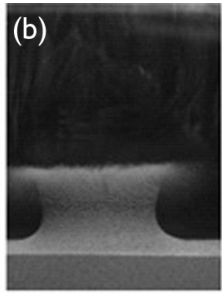

$t=33 \mathrm{~ms}$

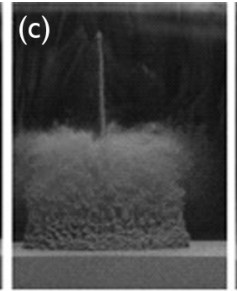

$t=108 \mathrm{~ms}$

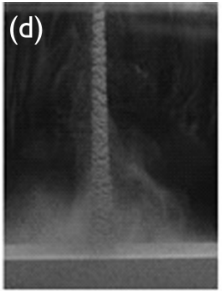

$t=233 \mathrm{~ms}$

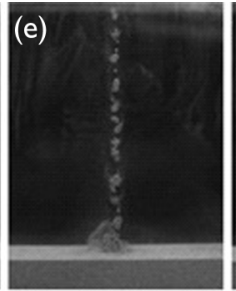

$t=452 \mathrm{~ms}$

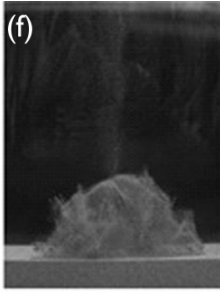

$t=583 \mathrm{~ms}$

(g)
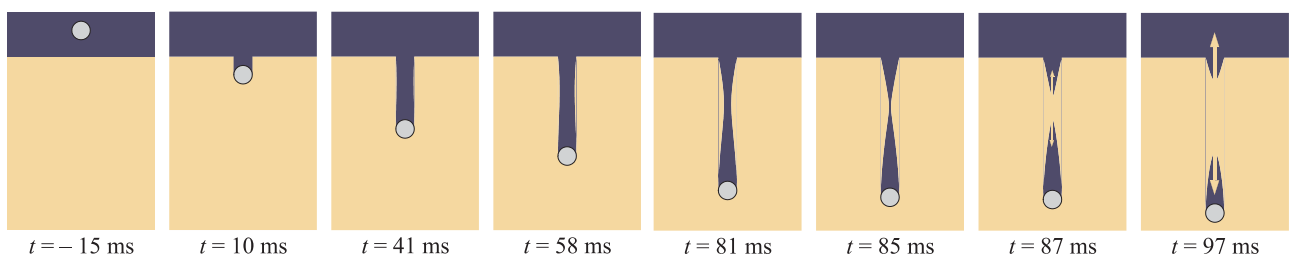

FIG. 12. (a)-(f) Impact (at $t=0 \mathrm{~s}$ ) of a steel ball (diameter $2.5 \mathrm{~cm}$ ) on soft decompactified sand (grain diameter typically $40 \mu \mathrm{m}$ ). The splash and the jet emerge, just as in water. The grains in the jet cluster due to their inelastic collisions. The last frame shows a granular eruption caused by the rising air bubble. (g) Cross section of the void collapse following from the axially symmetric Rayleigh-type model of Ref. [76]. The void is pressed together by the hydrostatic pressure from the side, leading to a singularity and an upward and downward jet. Both series of images have been taken from Ref. [76].

interaction, and finally (partial) drop evaporation or solidification. One of the most burning questions Océ had in those days was on the fluid dynamics in the printhead.

A schematic cross section through a typical modern microelectromechanical system (MEMS)based printhead is shown in Fig. 13(a). The piezoactuator, similar to the piezoactuator used to generate single-bubble sonoluminescence, gives short pulses with (depending on the printer) a frequency of around $20-100 \mathrm{kHz}$. Each pressure pulse drives out a little droplet. Altogether, typically there are presently hundreds of nozzles within one silicon chip and several chips are integrated in one printhead. A modern inkjet printer has tens of such printheads.

Hans Reinten then told me that, unfortunately, this very fast and precise printing facility can break down from time to time: After billions of cycles a distortion of the droplet formation in a channel can develop. Either this distortion vanishes after a short time [Fig. 13(b)] or the jetting process of that channel eventually completely breaks down. The only solution then is to turn off the piezoactuator and wait for a minute or so, which of course is extremely annoying for a high-speed printer (though other nozzles might take over during that time). The suspected culprit for the problem was a bubble within the inkjet channel. However, how does the bubble get there and what is its dynamics? Also, how is this trouble avoided?

To solve this problem, we employed the same method as for the snapping shrimp [69] or the entrained bubble at water droplet impact [68]: watch and listen. That is, we measured the acoustic response of the channel and combined it with high-speed imaging. Indeed, we found that the distortion of the droplet is correlated with a modification of the acoustic response of the channel. This result indeed suggested that the distortion originates from a bubble, because bubbles modify the acoustical behavior of the channel [94,95]. In fact, we were even able to "hear" the size of the bubble (see Fig. 14). However, how does the bubble get there? Is it nucleated or entrained at the nozzle?

By combining high-speed imaging for the inkjet and infrared imaging for the interior of the ink channel, we succeeded in visualizing how a bubble is entrained at the nozzle $[93,96]$ and what its dynamics inside the channel are [Fig. 13(c)]. Here small dirt particles, either on the nozzle plate or in the ink channel, play a crucial role. Once a tiny bubble is entrained at the nozzle, the acoustical forces pull it into the channel. Just as in single-bubble sonoluminescence, the oscillating bubble 
(a)

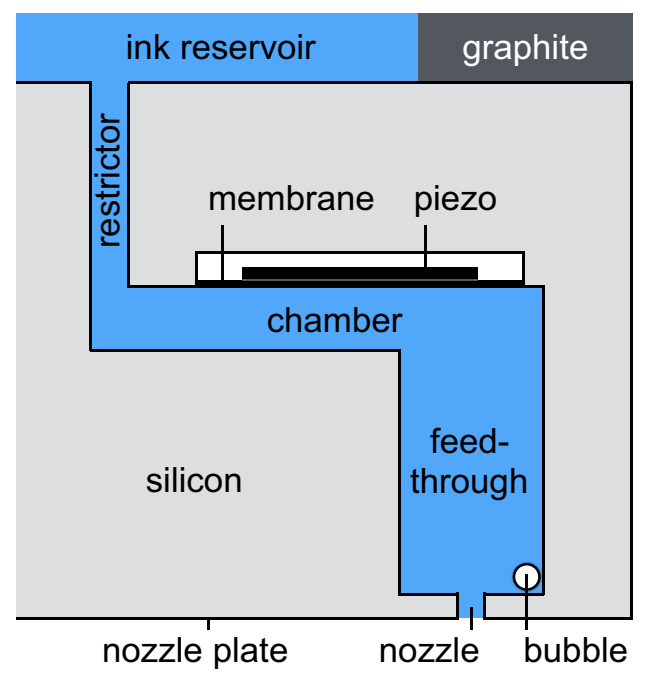

(c)

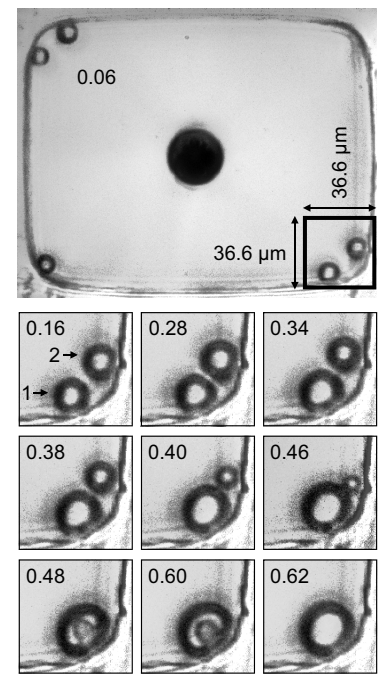

(b)

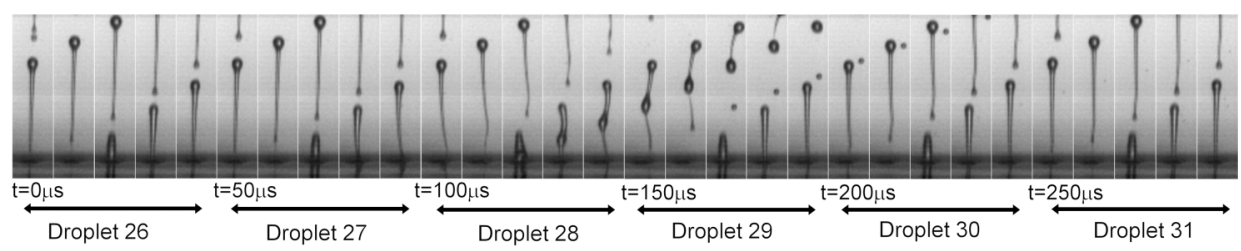

FIG. 13. (a) Schematic of the ink channel (side view) of a MEMS-based drop-on-demand inkjet printer. An air bubble has been entrained and pushed to the corner of the ink channel. (b) Droplet distortion (droplets 28 to 29) caused by a dirt particle around the jetting nozzle. Such a dirt particle can lead to bubble entrainment. In the case shown here the normal droplet formation process recovers and becomes regular again. The figure has been taken from Ref. [93]. (c) The top shows the infrared bottom view through the silicon around the nozzle into the ink channel. In the center the nozzle is seen. In three of the four corners bubbles got entrained which affect the printing process. The nine smaller images show the diffusive dynamics of the bubbles, clearly revealing bubble growth (by rectified diffusion) and Ostwald ripening. The times are given in seconds. The images have been taken by Arjan Fraters, Physics of Fluids group, Twente, in collaboration with Océ.

then grows in the acoustic field by rectified diffusion. So the knowledge which we had acquired from single-bubble sonoluminesence, namely, on acoustical forces on a bubble and on rectified diffusion, was essential in solving this problem.

Once the bubble has grown by rectified diffusion to considerable size, the actuation pressure pulse to jet a droplet simply leads to bubble compression, and not to a pressure increase at the nozzle. Therefore, no droplets can be jetted any longer. Only after the acoustic pressure has been turned off can the bubble dissolve by diffusion so that printing becomes possible again.

The final goal of course must be to avoid the entrainment of the bubble or to immediately get rid of it again, e.g., by applying an acoustical pulse immediately after the bubble has been detected. For the next step, the development of even faster printers with even smaller droplets, further fundamental work on the meniscus instability leading to the bubble entrainment remains essential.

Bubble entrainment also turned out to be crucial for so-called immersion lithography, pioneered by ASML, the world's leading supplier of lithography systems for the semiconductor industry. Once the photolithography of wafers had been at the edge of optical resolution, even smaller structures were realized by introducing lithography machines using immersion technology, i.e., lithography under a film of water (with a refractive index of 1.33, rather than 1.00 for air) between the wafer and optical lens [see Fig. 15(a)], allowing for smaller structures on the wafer than standard lithography 

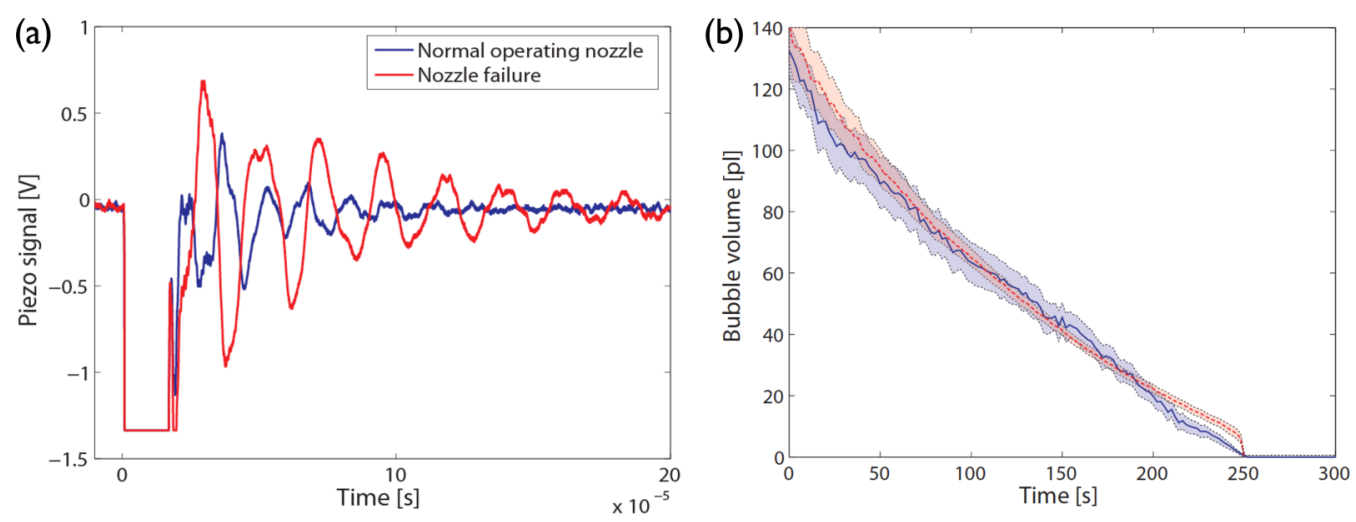

FIG. 14. (a) Acoustic response (as reflected in the piezocurrent) of a normally operating nozzle (blue line) and with an entrained air bubble with a volume of $V_{b}=80 \mathrm{pl}$ (red) close to the nozzle plate. It can be seen that the volume oscillations of the entrapped bubble modify the piezocurrent significantly: The piezocurrent amplitude is less damped and the main frequency decreases. (b) Comparison of the optically and acoustically measured bubble volume during bubble dissolution (which takes about $250 \mathrm{~s}$ here). The acoustically measured bubble volume is shown as a red dotted line and the optically measured bubble volume as a blue solid line. The areas around the lines give the error margins in the results. The figures have been taken from Ref. [94].

in air. However, the development of immersion lithography machines has been hindered by two fundamental fluid dynamical problems, namely, the entrainment of bubbles into the water and the loss of water from the film [see Fig. 15(b)]. Both of these contact line instabilities have challenged the further development of immersion lithography, as obviously the entrainment of light-scattering

(a)

(b)

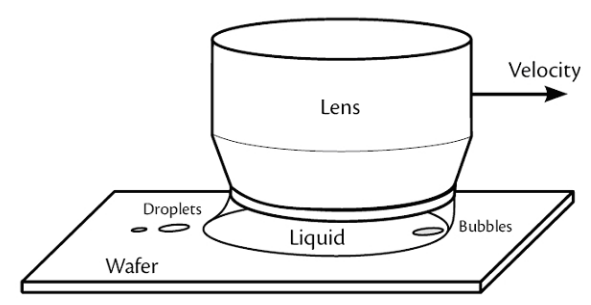

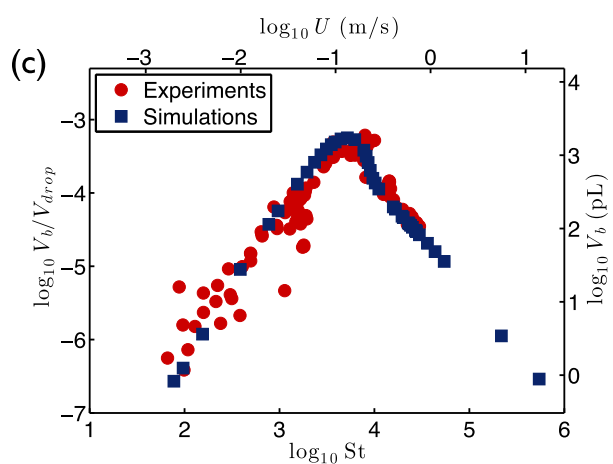

(d)

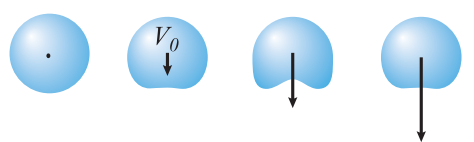

FIG. 15. (a) Schematic cross section of an immersion lithographic scanning device. The water in between the lens and the wafer reduces the imaging resolution below $40 \mathrm{~nm}$. (b) Visualization of the contact line instabilities. Bubbles are entrained at the advancing contact line, whereas droplets are lost at the receding contact line. (c) Entrained bubble volume after drop impact versus impact velocity, given in both dimensional units (right and top axis) and dimensionless units (left and bottom axis), showing a clear maximum. To the left capillarity prevails, while to the right inertia prevails. Red circles correspond to experiments with color interferometry, blue squares to boundary integral simulations coupled to a viscous lubrication approximation. The figure has been adopted from Ref. [97]. (d) Sketch of the droplet impact and bubble entrainment mechanism, with (from left to right) increasing velocity. 
bubbles into the film or the partial loss of the film is unacceptable for the lithography process. These hydrodynamic instabilities set in at a certain velocity with which the wafers under the lens are pulled away. It is thus this hydrodynamic instability which sets the production rate of the wafers and therefore the price of the lithographic system.

In the context of this problem, together with ASML, we looked at the entrainment of bubbles under droplets impacting on a solid substrate, following pioneering work of the Chicago, Harvard, Kaust, and other groups [98-103] (for recent reviews, see Refs. [104,105]). By combining highspeed color interferometry [106], scaling arguments, and numerical simulations with the boundary integral method for the droplet coupled to a viscous lubrication approximation for the gas flow in the thin and narrowing gap between impacting droplet and substrate, we found that there is an optimal velocity for maximal bubble entrainment [97]: For lower velocities the impacting droplet remains more spherical (capillary regime) and for higher velocity (inertial regime) the drop is smashed against the surface so much that not much air can be entrained either [see Fig. 15(c)]. Obviously, this work is also of interest for the inkjet industry and the coating industry.

\section{FROM SURFACE BUBBLES TO SONOCHEMISTRY AND ULTRASONIC CLEANING}

As stated above, single-bubble sonoluminescence can be seen as the hydrogen atom of bubble fluid dynamics. However, atomic physics did not stop with the understanding of the hydrogen atom but moved ahead to more complicated and interacting atoms, molecules, and condensed matter. So it was also our desire to better understand interacting bubbles, but in the most controlled way, with fixed distance. To achieve this acoustically is difficult, as acoustically driven microbubbles either repel or attract each other through the so-called Bjerknes forces of the second kind [1,107], depending on their size and the driving pressure. If trapped by the primary Bjerknes forces in different pressure antinodes of the acoustic field, they are too far away $(7.5 \mathrm{~cm}$, corresponding to the
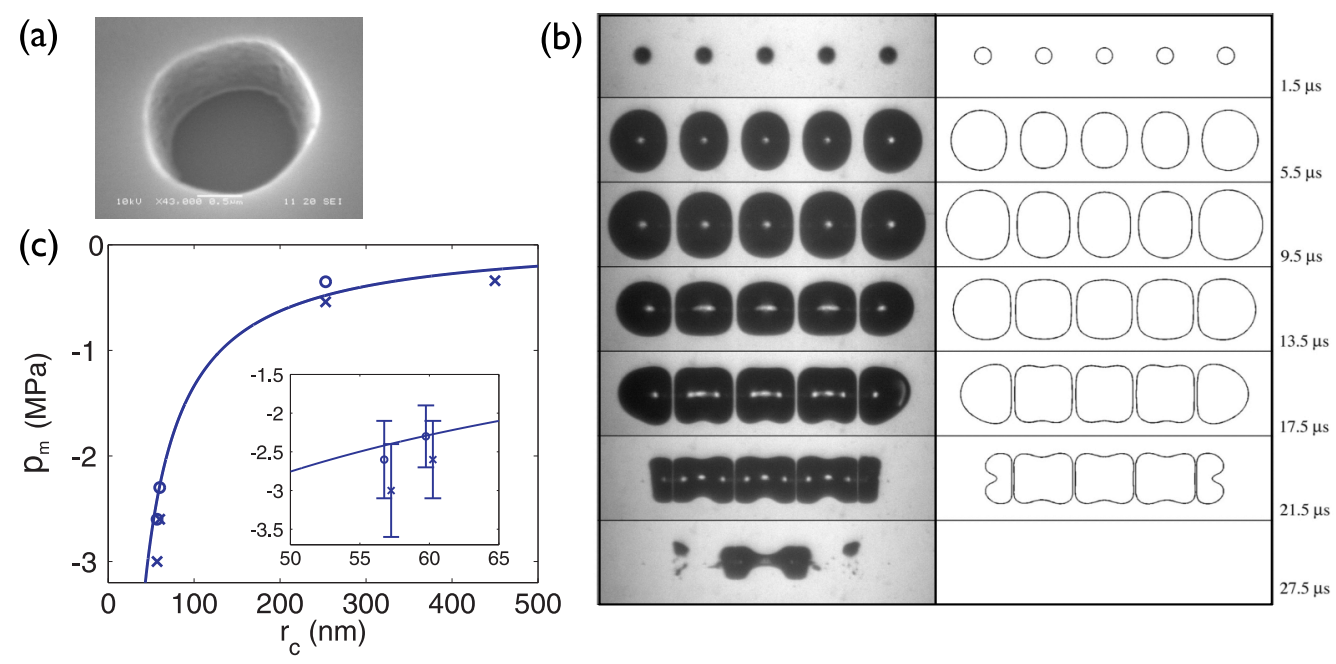

FIG. 16. (a) Electron microscopy image of an individual hydrophobic microcavity (diameter $4 \mu \mathrm{m}$ ) etched on a silicon plate acting as the gas trap. The photo has been taken from Ref. [109]. (b) Comparison between experiment and boundary integral simulation of the cavitation of five bubbles in microholes set on a line with a distance of $d=200 \mu \mathrm{m}$ and a driving pressure of $P_{a}=-1.4 \mathrm{MPa}$. One can clearly see the shielding effect for the inner bubble, collapsing later than the outer ones. The figure has been taken from Ref. [109]. (c) Nucleation threshold $p_{m}$ as a function of the pit radius $r_{c}$ for both theory (line) and experiment (crosses represent nucleation and circles no nucleation). The inset shows a zoom in with error bars. For visibility, overlapping points are shifted $\pm 0.25 \mathrm{~nm}$ with respect to each other. The figure has been taken from Ref. [110]. 
acoustic wavelength for $f=20 \mathrm{kHz}$ in water) from each other to considerably interact. Therefore, we came up with the idea to trap the bubbles through pinning forces to hydrophobic micromachined microcavities which act as gas traps when the substrate is immersed in water [108,109] [see Figs. 16(a) and 16(b)]. The patterning of the substrate allows us to control the number of bubbles and the distance between them. Each hemispherical bubble experiences the effect of its mirror image. Correspondingly, an isolated hemispherical bubble together with its mirror image behaves like a free spherical bubble, i.e., its dynamics is well described by the Rayleigh-Plesset equation (1). By putting the microcavities close to each other, we could study interacting microbubbles, either in a row (i.e., in one dimension) as in Fig. 16(b), where we could compare the results with boundary integral simulations (i.e., under the potential flow approximation) with axial symmetry, or in two dimensions with bubbles arranged on a surface in any order.

In fact, by varying the diameter of the microhole down to $100 \mathrm{~nm}$ and less, we could quantitatively test [110] the crevice model of bubble nucleation [111-114]. Figure 16(c) compares the nucleation threshold calculated from the crevice model [114] with the experimental data, finding good agreement. We also used such hydrophobic micromachined pits as artificial crevices for bubble nucleation to achieve higher sonochemical yields at ultrasound powers that would otherwise not produce a significant chemical effect [115] and for ultrasonic cleaning purposes [116], in both cases making use of the energy focusing power of the collapsing bubbles. Out of this activity another spin-off company emerged from our group. Finally, with such pits we enhanced the heat flux in thermal convection by vapor-bubble nucleation [117].

\section{FROM SURFACE NANOBUBBLES TO CATALYSIS AND ELECTROLYSIS}

Being interested in tiny surface bubbles, the so-called surface nanobubbles caught my attention, which from about 2000 on have been found in atomic force microscopy (AFM) images of water-immersed, preferentially hydrophobic substrates [118-120] [see Fig. 17(a)]. One of course immediately wonders why such surface nanobubbles are stable. Because of the diverging Laplace pressure $p_{\text {Laplace }}=2 \sigma / R$, where $\sigma$ is the surface tension and $R$ the radius of curvature, tiny bubbles should dissolve immediately: In water the gas pressure inside a bubble with $R=10 \mathrm{~nm}$ is $p_{\text {gas }}=P_{0}+p_{\text {Laplace }} \approx 145$ atm. With Henry's law this translates to a gas concentration at the edge of the bubble $c_{R}=p_{\text {gas }} c_{s} / P_{0}$ which is 145 times larger than the saturation concentration $c_{s}$ and therefore to a large concentration gradient away from the bubble, leading to very fast dissolution. This even holds in the case of (slight) gas oversaturation, i.e., as long as the gas oversaturation

$$
\zeta=\frac{c_{\infty}}{c_{s}}-1
$$

(a)

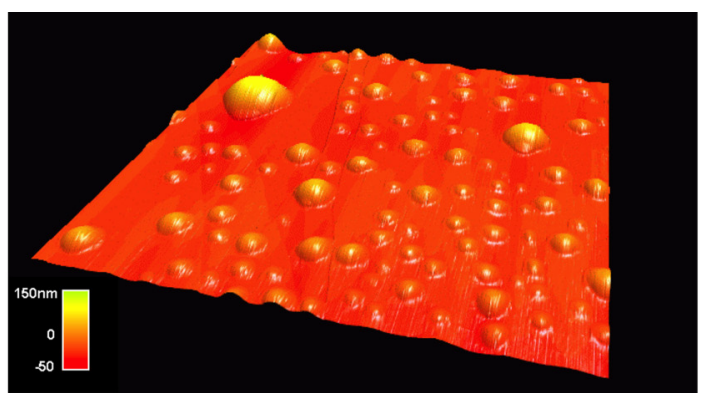

(b)

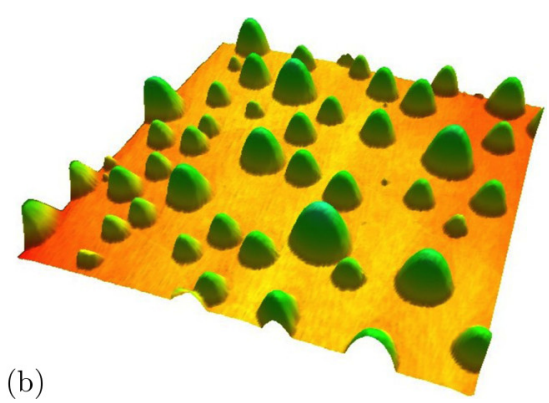

FIG. 17. (a) The AFM image $\left(4 \times 4 \mu \mathrm{m}^{2}\right)$ of a surface nanobubble on a HOPG surface, obtained through the solvent exchange process. (b) The AFM image $\left(30 \times 30 \mu \mathrm{m}^{2}\right)$ of surface nanodroplets on a hydrophobically coated Si surface, also obtained through the solvent exchange process. The color code goes from 0 (red) to $800 \mathrm{~nm}$ (green). Figures have been taken from our recent review article on surface nanobubbles and surface nanodroplets [121]. 
is not too large. In the above-mentioned classical paper by Epstein and Plesset [20], known to us from our work on single-bubble sonoluminescence, the dissolution time of such a bubble had been calculated from the diffusion equation and the corresponding boundary conditions. The result for the typical dissolution timescale is $\tau_{E P}=R_{0}^{2} \rho_{g} / 2 c_{s} D$, where $D$ is the diffusion constant and $\rho_{g}$ the gas density. For the bubble with $R=10 \mathrm{~nm}$ and the material constants for water, one indeed gets $\tau_{E P} \approx 3 \mu$ s, i.e., such nanobubbles should dissolve basically immediately.

The surface nanobubbles in the AFM image in Fig. 17(a) were generated with the so-called solvent exchange process, which had been pioneered by Xuehua Zhang et al. [122,123]. Here a gas-saturated liquid having high gas solubility (e.g., ethanol) is replaced by another liquid with lower gas solubility, e.g., with water. This leads to a local gas supersaturation $\zeta>0$ and therefore to the nucleation of bubbles. Macroscopically, we know this effect of everyday life: When we fill a water glass with cold tap water and leave it for a while in a warm room, small air bubbles form on the inside of the glass. The reason is that in general tap water is oversaturated with air and gases in cold water dissolve much better than in warm water. If the tap water in the glass slowly warms up to room temperature, the gas solubility is reduced and bubbles nucleate on the edge of the glass. Depending on the size of the glass, the bubbles last about four days, as anyone can easily try.

First it was speculated that the stability of surface nanobubbles is due to surfactants [124], which, however, for various reasons (explained in Ref. [121]) could be ruled out. Moreover, with the help of fluorescence lifetime imaging microscopy it could be shown [125] that the objects observed with AFM are indeed air bubbles and not nanodroplets of a contaminating liquid. In addition to the remarkable stability of the nanobubbles, another paradox was that their contact angle (measured on the bubble side) was not Young's angle, as known from macroscopic measurements, but much smaller [126,127].

When there is such a large gap between experiment and theory, numerical simulations often help. We therefore performed molecular-dynamics (MD) simulations of surface nanobubbles [128], and at least those adhered to the theoretical expectation: They dissolved in microseconds.

The key to solving this paradox came from the experiments of Xuehua Zhang et al. [129], who observed that surface nanobubbles exposed to gas-undersaturated water $(\zeta<0)$ dissolve slowly, but (initially) not by reducing their lateral size, but by reducing their contact angle (on the gas side) [see Fig. 18(a)]: The three-phase contact line remains pinned. This dissolution mode is called CR mode, standing for constant contact radius, in contrast to the so-called CA mode, standing for constant contact angle [130,131]. Pinning dramatically changes the dissolution scenario: The Laplace pressure $p_{\text {Laplace }}=2 \sigma / R$ now no longer diverges, but approaches zero [see Fig. 18(b)]. Thus, when the bubble dissolves, no large internal pressure can build up and thus there is no concentration gradient from the outside of the bubble to the predetermined concentration level $c_{\infty}$ far away from the bubble: The bubble becomes stable.

The reason for the pinning lies in the unavoidable surface inhomogeneities of geometric and/or chemical nature. These are also relevant in the above-mentioned daily life phenomenon of bubble formation in a glass with cold tap water which warms up or when we pour soda water into a glass: In both cases bubbles nucleate out of oversaturated water on such inhomogeneities. Macroscopically, the surface inhomogeneities lead to contact line hysteresis, as studied extensively by de Gennes and co-workers in the 1990s [132,133].

We could generalize the classical Epstein-Plesset calculation [20] to calculate the diffusive dynamics of pinned surface bubbles [134]. Here the key idea was to adopt the quasistatic calculation of Popov [135] for the so-called coffee stain problem [136,137], which is on the evaporation of a liquid drop on a plain substrate. This is not surprising because both processes are controlled by diffusion outside of the drop or bubble: in the evaporating droplet case, of diffusion of water vapor in air, and in the surface nanobubble case, of air into water. For bubbles with constant contact diameter $L$ (i.e., in the CR mode), the result of this adopted (quasistatic) calculation reads [134]

$$
\frac{d \theta}{d t}=-\frac{4 D}{L^{2}} \frac{c_{s}}{\rho_{g}}(1+\cos \theta)^{2} f(\theta)\left[\frac{L_{c}}{L} \sin \theta-\zeta\right],
$$


(a) unpinned: "CA-mode"

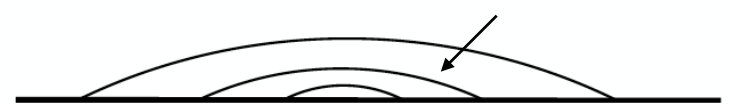

pinned: "CR-mode"

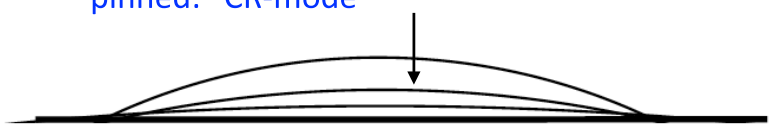

(c)

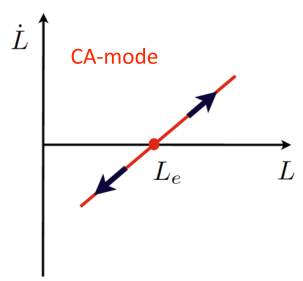

$\dot{\theta}$

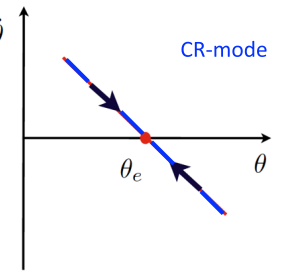

(b)

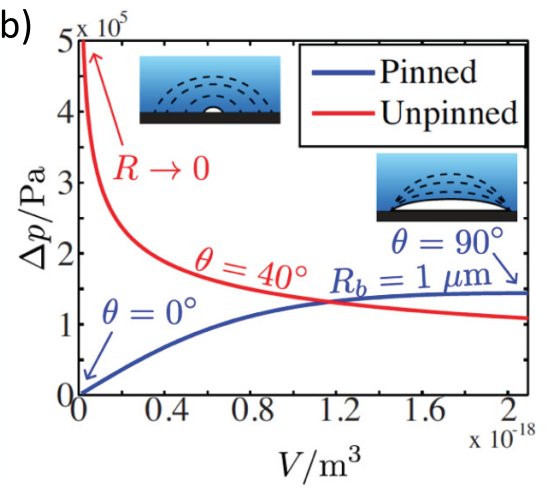

(d)

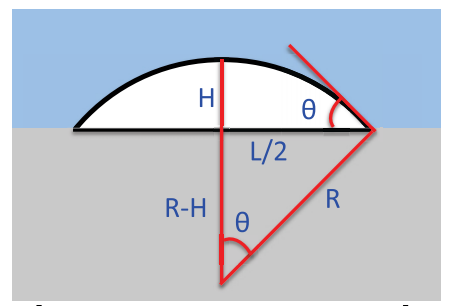

FIG. 18. (a) The nanobubbles can dissolve in two different modes (the time direction is indicated by the arrow): In the CA mode (constant contact angle, top), the contact angle is constant. As a result, the radius of curvature becomes smaller and smaller, which leads to the divergence in the Laplace pressure, as can be seen in (b) (red curve), where we show $\Delta p=p_{\text {Laplace }}$ as a function of the bubble volume $V$. In the CR mode (constant contact radius, bottom), the contact radius is constant and the contact angle becomes smaller. As a result, the radius of curvature increases in the course of the dissolution process and the divergence in Laplace pressure does not occur [blue curve in (b)]. In (c) we show a sketch of the phase space for the stable equilibrium, which results in pinning (CR mode, bottom), with the equilibrium contact angle $\theta_{e}$, given by Eq. (8), and a sketch of the phase space for unstable equilibrium without pinning (CA mode), in which the surface bubble either shrinks or grows. In (d) the notation used is introduced: $L$ is the lateral extent of the bubble at the substrate (contact diameter), $H$ is the bubble height, $\theta$ is the contact angle at the gas side, and $R$ is the radius of curvature.

with a positive definite $f(\theta)$ given in Ref. [135] and a critical lateral extension $L_{c}=4 \sigma / P_{0} \approx$ $2.84 \mu \mathrm{m}$ for air bubbles in water with $1 \mathrm{~atm}$ ambient pressure. From Eq. (7) it immediately follows that for gas undersaturation $-1 \leqslant \zeta<0$ no stable surface nanobubbles can exist, as then the righthand side of Eq. (7) is always negative: The bubble dissolves down to $\theta=0$. For gas oversaturation $\zeta>0$, however, a stable equilibrium with the equilibrium contact angle [134]

$$
\sin \theta_{e}=\zeta \frac{L}{L_{c}}
$$

can exist. That the equilibrium indeed is stable is seen from the phase space in Fig. 18(c), bottom. In this stable equilibrium, Laplace pressure (causing gas flux out of the bubble) and gas overpressure (causing gas influx) are in balance.

From Eqs. (7) and (8) we also see that for too large oversaturation $\zeta>L_{c} / L$ there is no stable equilibrium and the surface bubble keeps on growing so that it will finally detach. The condition $L<L_{c} / \zeta$ is the reason that there can be stable surface bubbles only on a microscopic scale. The 
(a)
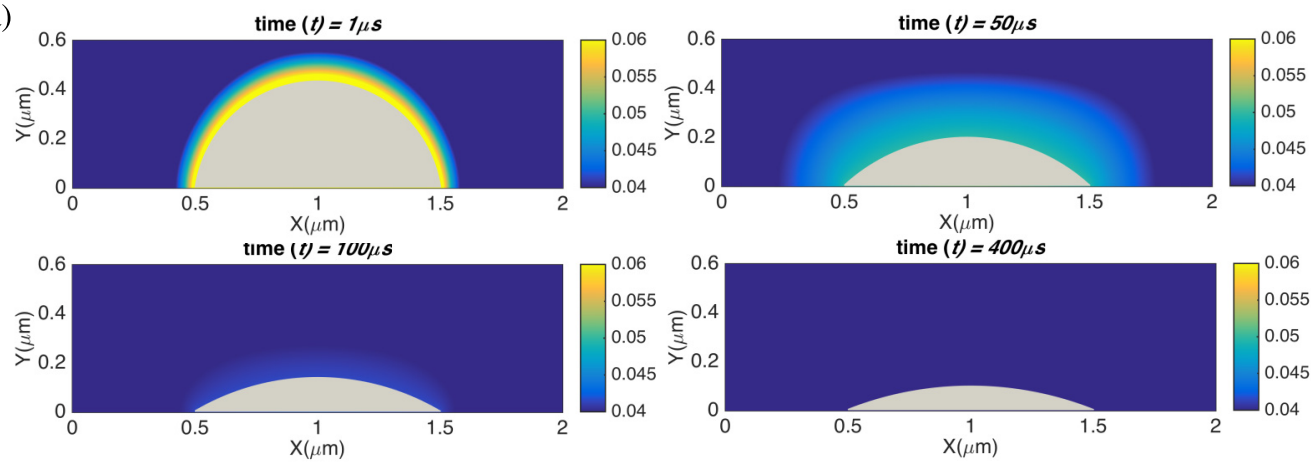

(b)

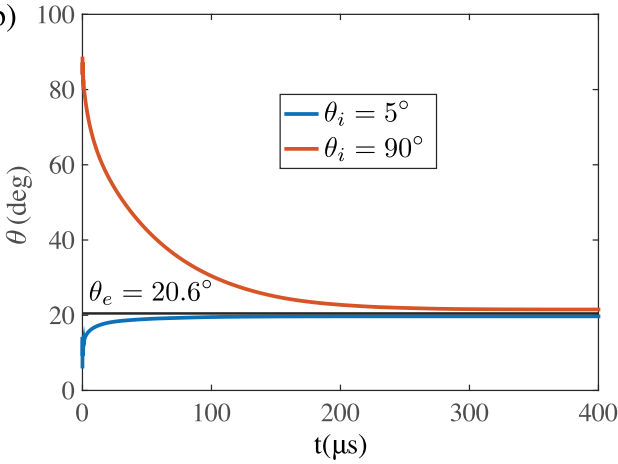

(c)

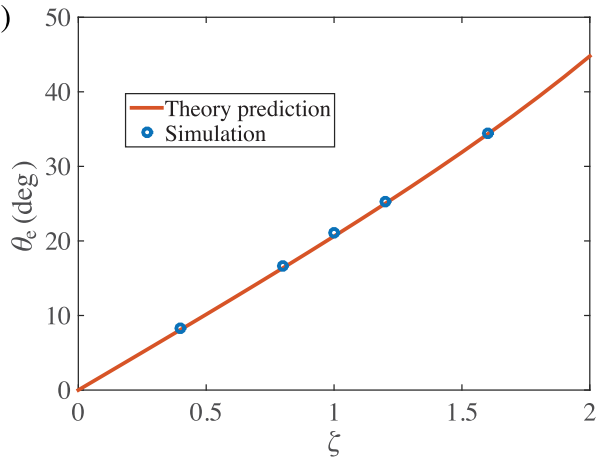

FIG. 19. Results from a numerical simulation (using the finite-difference method) of the diffusion equation, with the shrinking or growing pinned surface bubbles coupled with the immersed boundary method. (a) Snapshots of the diffusive dynamics of a pinned surface nanobubble growing towards its equilibrium state. The color code represents the gas concentration field. Here $L=1 \mu \mathrm{m}$ and $\zeta=1$. (b) Time evolution $\theta(t)$ of the contact angle growing or shrinking towards its equilibrium value $\theta_{e}$ given by Eq. (8). Two cases with different initial contact angles $\theta_{i}$ are shown. Here $L=1 \mu \mathrm{m}$ and $\zeta=1$. (c) Equilibrium contact angle $\theta_{e}$ for various gas concentrations $\zeta$. The straight line is the prediction (8), giving perfect agreement. Again, $L=1 \mu \mathrm{m}$. In the simulations here, the domain size is $6 \times 3 \times 6 \mu \mathrm{m}^{3}$. The figures have been taken from Ref. [138].

stabilization mechanism does not work on a macroscopic scale because then the Laplace pressure is too weak and cannot compensate for the gas overpressure from the outside. We also see that on the microscopic scale, the radius of curvature of a surface bubble is not given by Young's equation but by the relationship (8). We note that Eq. (8) and the stability of the equilibrium have also been confirmed both by numerical simulations of the full diffusion equation [138], employing immersed boundary methods for the growing or shrinking bubble (see Fig. 19), and also by MD simulations, but now with built-in pinning [139] (see Fig. 20).

As we see, the size $L$ of the pinning site and the oversaturation $\zeta>0$ determine the stability of the surface nanobubbles. However, how is the oversaturation $\zeta$ determined? When the liquid container with the surface nanobubbles on some substrate is closed and in equilibrium, $\zeta$ remains constant, hence the equilibrium contact angle $\theta_{e}$ [Eq. (8)]. In an open vessel, on the other hand, an initial gas oversaturation $\zeta>0$ will not last long due to diffusive processes with the outside world [140]. If the distance to the outside world is $\ell$, the typical diffusive timescale is $\tau_{\text {outer }} \sim \ell^{2} / D$. For $\ell=1 \mathrm{~cm}$ we get $\tau_{\text {outer }} \approx 14 \mathrm{~h}$ and for $\ell=3 \mathrm{~cm} \tau_{\text {outer }} \approx 5 \mathrm{~d}$ : This is exactly the timescale that we also observe for the dissolution process of air bubbles which nucleate at the edge of the above-mentioned glass filled with cold, gas-supersaturated tap water when put into a warmer room.

Just as single-bubble sonoluminescence can be seen as the hydrogen atom of inertial bubble dynamics [15], a single surface nanobubble can be seen as the hydrogen atom of diffusive bubble dynamics. Its properties, in particular its on first sight surprising stability and its small contact angle, 
(a)

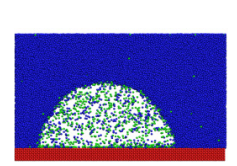

$t=1.75$ ns

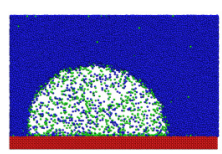

$\mathrm{t}=3.5 \mathrm{~ns}$

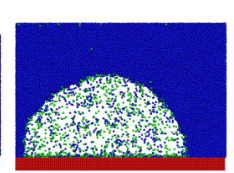

$t=5.25 n s$

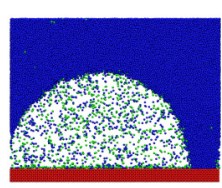

$\mathrm{t}=7.0 \mathrm{~ns}$

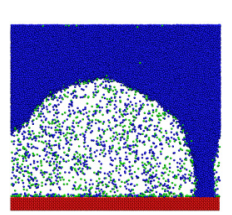

$t=8.75 n s$

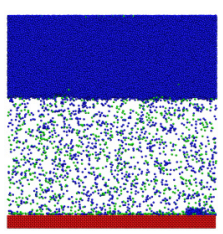

$t=9.625 n s$

(b)

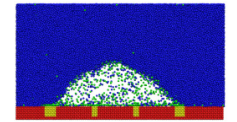

$t=1.05 \mathrm{~ns}$

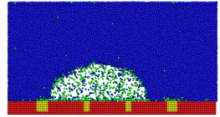

$t=2.8 n s$

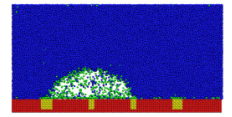

$t=4.9 \mathrm{~ns}$

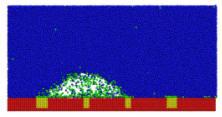

$t=14.0 \mathrm{~ns}$

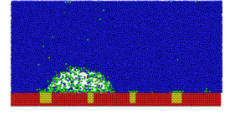

$t=25.2 \mathrm{~ns}$

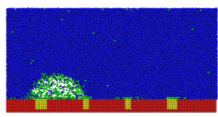

$\mathrm{t}=31.5 \mathrm{~ns}$

(c)

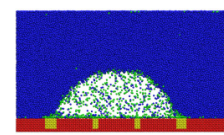

$\mathrm{t}=0.0 \mathrm{~ns}$

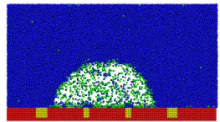

$\mathrm{t}=1.05 \mathrm{~ns}$

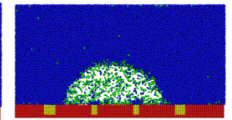

$\mathrm{t}=4.9 \mathrm{~ns}$

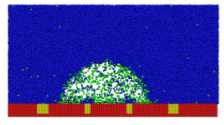

$t=14.0 \mathrm{~ns}$

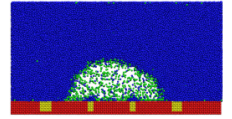

$t=25.2 n s$

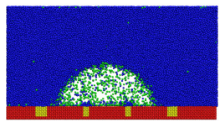

$\mathrm{t}=31.5 \mathrm{~ns}$

(d)

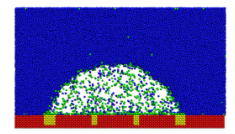

$t=1.75 n s$

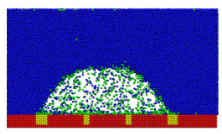

$\mathrm{t}=3.5 \mathrm{~ns}$

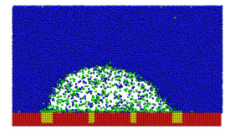

$\mathrm{t}=7.0 \mathrm{~ns}$

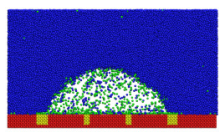

$t=14.0 \mathrm{~ns}$

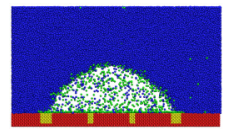

$t=21.0 \mathrm{~ns}$

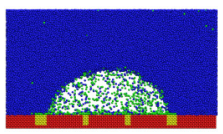

$t=31.5 n s$

FIG. 20. Time evolution of a surface nanobubble in MD simulations. (a) Without chemical heterogeneities and gas-oversaturated liquid $(\zeta>0)$ the bubble grows. (b) With hydrophobic chemical heterogeneities and gas-undersaturated liquid $(\zeta<0)$ the bubble shrinks. (c) With hydrophilic chemical heterogeneities and gas-oversaturated liquid $(\zeta>0)$ the pinning force for nanobubble stabilization is not sufficient. (d) Only with hydrophobic chemical heterogeneities and gas-oversaturated liquid $(\zeta>0)$ does one get a stable surface nanobubble. The figure has been taken from Ref. [139].

are meanwhile reasonably well understood (see our review article in Ref. [121]). As the next step, we have moved towards diffusively interacting surface nanobubbles. Just as for inertial bubbles [Sec. VII and Fig. 16(b)], one can best study also the diffusive behavior of surface bubbles by fixing their distance by offering "weak spots" on a hydrophobic surface, namely, by micromachining the surface: During the solvent exchange, the bubbles will nucleate in the cavities and grow [141]. In Fig. 21(a) we show snapshots of this growth process over a period of $3 \mathrm{~min}$. Note the very different timescale as compared to the inertial bubble dynamics of Fig. 16(b), where the whole series of snapshots is less than $25 \mu \mathrm{s}$.

For interacting surface nanobubbles, according to above-sketched theory [134], one would expect that in equilibrium all surface nanobubbles would have the same radius of curvature $R_{e}=L_{c} / 2 \zeta$. On first sight, one may expect that this equilibrium is unstable due to Ostwald ripening of the bubbles [145]: Small bubbles shrink due to their larger Laplace pressure and neighboring larger ones grow. However, it turns out that again it is pinning which stabilizes these neighboring bubbles against Ostwald ripening [146,147].

As stated above, a central idea of solving the surface nanobubble paradox originated from the analogy to the pinned coffee stain [136]: The first problem is controlled by diffusion of air in liquid, the latter one by diffusion of vapor in air. In between these two cases is the one of a liquid surface nanodroplet [see Fig. 17(b)] in a sparsely miscible host liquid, for which the stability (with pinning, or lack thereof, without pinning) is given by the same equations and mechanisms [121]. 
(a)

$0 \mathrm{~s}$

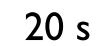

$66 \mathrm{~s}$

$120 \mathrm{~s}$

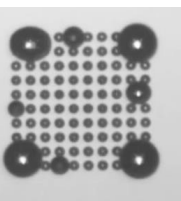

$180 \mathrm{~s}$

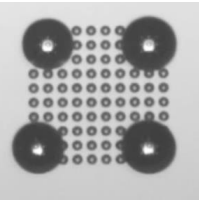

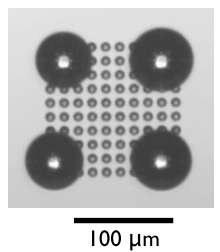

(b)
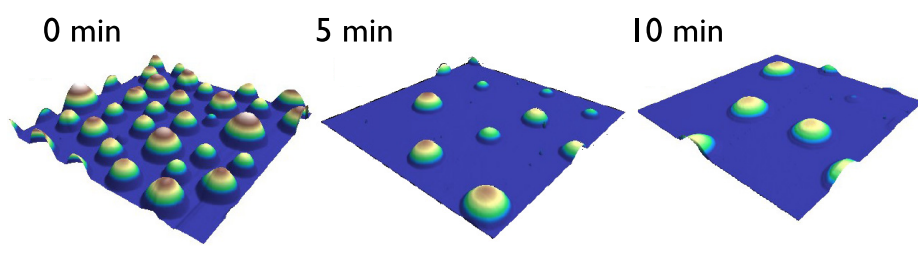

$20 \mathrm{~min}$

FIG. 21. (a) Controlled microbubble formation and growth on hydrophobic surfaces decorated with micromachined micropits (with a diameter of $10 \mu \mathrm{m}$ and edge-to-edge distance of $13 \mu \mathrm{m}$ ) by solvent exchange. The microchannel was first filled with air-saturated water, which was then replaced by ethanol solution under a flow rates of $100 \mu \mathrm{L} / \mathrm{min}$. The figure has been taken from Ref. [142]. (b) Dissolution process of an ensemble of HDODA nanodroplets with a typical contact diameter of $L=10-30 \mu \mathrm{m}$. The color scale corresponds to a height of $0 \mu \mathrm{m}$ (blue) to $0.9 \mu \mathrm{m}$ (red) of the droplets. The dissolution process was stopped by polymerization of the HDODA by UV radiation, so a visualization of the droplets by AFM became possible, a trick introduced in Ref. [143]. The figure has been taken from Ref. [144].

Not only are the dissolution processes of such surface nanodroplets [see Fig. 21(b)] completely analogous to those of surface nanobubbles, but also the nucleation and growth processes. In particular, it is again the solvent-exchange process that allows surface nanodroplets to be generated in a controlled manner $[143,148]$ : Now a high-solubility liquid saturated with a certain substance is replaced by another with less solubility. The emerging oversaturation leads to droplet nucleation.

As an example, consider the Greek liquor ouzo [alternatively pastis (France), sambuca (Italy), or raki (Turkey)]: Ouzo consists of alcohol, water, and anise oil, which is very soluble in alcohol. When served, the ouzo is diluted with water. As a result, the alcohol concentration and thus the solubility of the oil decreases: Oil droplets then nucleate and grow in the supersaturated solution. This leads to the known milky appearance of the ouzo-water mixture. In the presence of a hydrophobic surface, nucleation naturally takes place at this surface and surface nanodroplets are formed in complete analogy to the surface nanobubbles. With the help of the control techniques of microfluidics, one can study very precisely the dependences of the nucleation process on the control parameters such as flow velocity, channel thickness, oil concentration, size and shape of the pinning sites, etc., and understand them theoretically and quantitatively [148-150].

The technological relevance of surface bubbles and droplets and the solvent-exchange process can hardly be underestimated. In our review article [121] we have dedicated a whole chapter to it; here I restrict myself to some particularly relevant and beautiful examples.

Liquid-liquid extraction, the transfer of a substance from one solvent to another, is one of the key processes of chemical technology and analysis. Since the work of Nobel Prize winner Fritz Pregl [151], efforts have been made to further miniaturize processes for chemical analysis and chromotography. The need to do this has increased in recent years [152]. First, it is important to be able to demonstrate ever smaller traces of chemical substances, be it in a medical context or for environmental reasons. Moreover, this proof must be fast and small samples should be sufficient, also to come to a "greener" chemical technology. One way to achieve this is single drop microextraction, where a substance A dissolved in water slowly dissolves in a drop of an organic solvent with a much higher solubility for A (e.g., carbon tetrachloride) held in the water. The drop is then extracted and analyzed. However, this process generally takes a long time and the efficiency is low. 
(a)
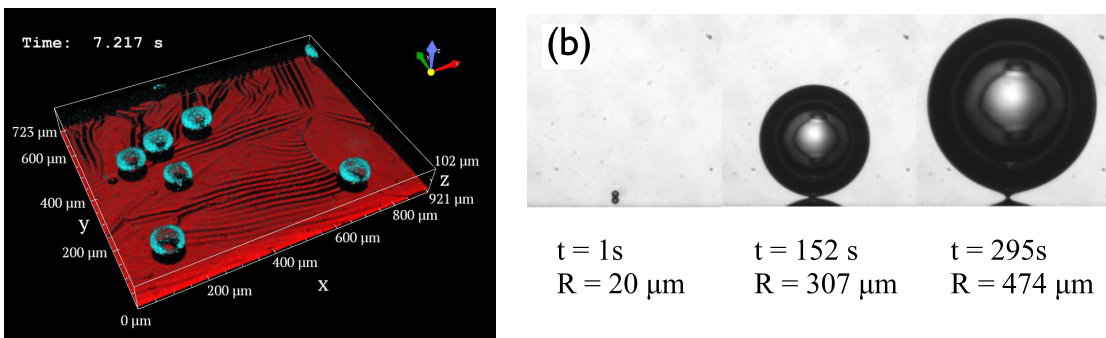

FIG. 22. (a) Confocal images of surface oxygen bubbles in a $\mathrm{H}_{2} \mathrm{O}_{2}$ solution ( $0.1 \%$ in water), growing on a (catalytic) platinum surface, $7.2 \mathrm{~s}$ after exposure of the platinum with the peroxide. The image was made by Pengyu Lv, Physics of Fluids, University of Twente. (b) Three snapshots of the same surface bubble, pinned at a micropit and growing out of a $\mathrm{CO}_{2}$-oversaturated solution with a (pressure controlled) oversaturation of $\zeta=0.18$. The figure has been taken from Ref. [162].

These limitations were elegantly overcome with the so-called disperse liquid-liquid microextraction (DLLME) technique [153-155] invented just 12 years ago. The technique involves a third liquid that is miscible with water and with carbon tetrachloride, e.g., ethanol. If a mixture of carbon tetrachloride and ethanol is now added to the water containing the substance A to be extracted, instantaneously many nano- and microdroplets of carbon tetrachloride nucleate, due to the enhanced water concentrations these molecules experience and the resulting lower solubility. With their very large total surface area, these nano- and microdroplets are very efficient to extract the substance A. As a final step, the carbon tetrachloride microdroplets are centrifuged off. The highly enriched substance A can then easily be detected by conventional methods. So far, DLLME is mostly optimized by trial-and-error; however, to achieve further control and progress, from my point of view it is very promising to apply the well-controlled methods of microfluidics and fluid dynamics to this process [156].

A good understanding and control of the diffusive processes in and around microdroplets and microbubbles may even save lives: In open-heart surgery, the bloodstream is decoupled from the heart and maintained by a heart-lung machine [157] and at the same time the blood is cooled down. In the cold blood more air dissolves than when it is warm. However, warming up the patient after surgery can be seen as a solvent-exchange process that can result in the nucleation of (surface) nanoand microbubbles that may significantly affect the blood supply locally. Here too it is essential and possibly lifesaving to well understand bubble nucleation in diffusive processes.

Further examples are electrolysis and catalysis: In both processes, the forming surface nano- and microbubbles considerably impair the efficiency, as they cover the electrode or catalyst. How does one control the growth of the bubbles and how to get rid of them? We have taken several initial steps to answer these questions [158-161]. Figure 22(a) shows catalytically grown oxygen bubbles on a platinum surface immersed in a hydrogen peroxide $\left(\mathrm{H}_{2} \mathrm{O}_{2}\right)$ solution. The platinum acts as a catalyst which triggers the decay of the peroxide to oxygen (evolving in bubbles) and water. For such bubbles evolving out of chemical reactions the relevant dimensionless number coming into play is the Damköhler number $\mathrm{Da}$, which is the ratio between the chemical reaction rate and the (diffusive or convective) mass transport rate. If $\mathrm{Da}$ is very large, the chemical reactions is controlled by the latter.

An example of a carbon dioxide bubble which is purely growing by diffusion is shown in Fig. 22(b). As discussed above, according to the Epstein-Plesset theory [20], for such bubbles the radius should grow in time with a square-root behavior. As we found in Ref. [162], in the beginning it indeed does, but later on convective effects take over, as the dissolved carbon dioxide affects the density of water. We also found that in the long term gas depletion effects become very relevant and bubbles nucleating later at the same location grow much slower than the earlier ones [163]. After growth, the bubbles finally detach. This happens because of buoyancy, either directly once 


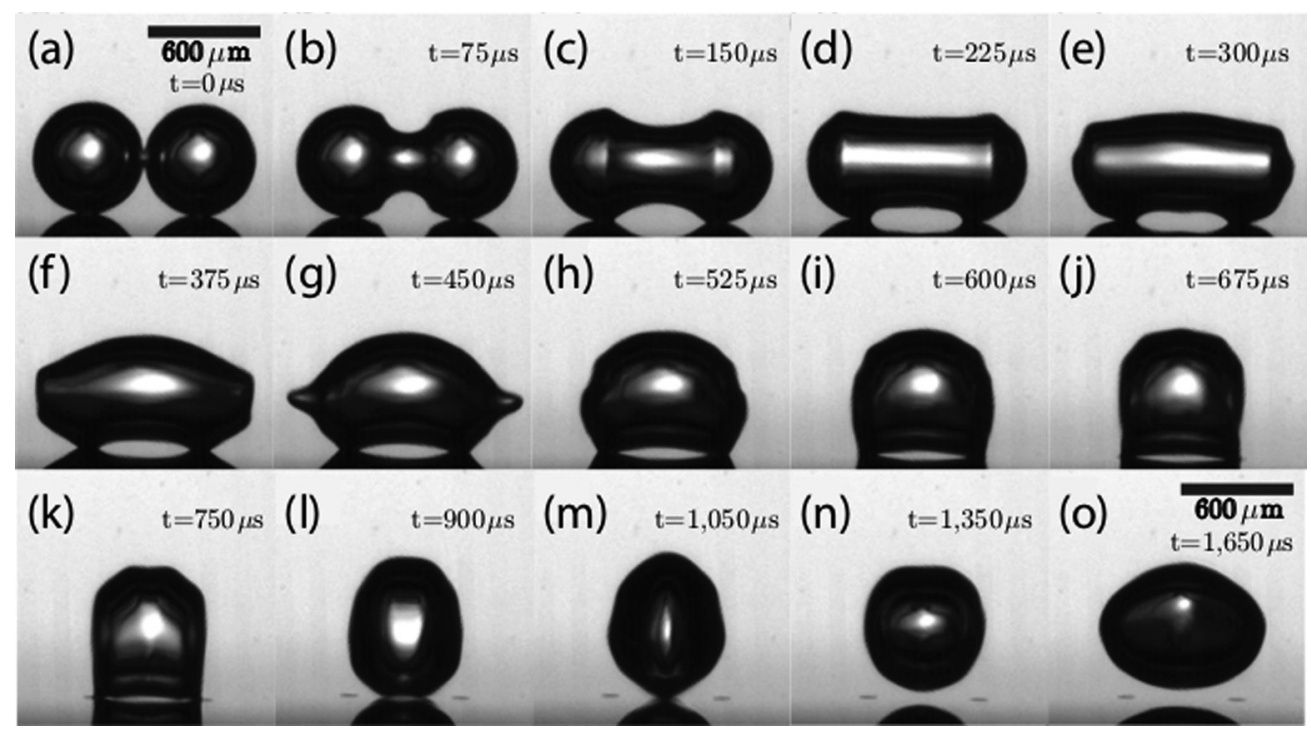

FIG. 23. Snapshots of the coalescence of two diffusively grown gas bubbles pinned to 50- $\mu$ m-radius pits. When they touch and coalesce, both bubbles have a diameter of $600 \mu \mathrm{m}$. Three main stages can be identified: (a)-(d) neck formation between the two bubbles, (e)-(l) propagation of the capillary waves along the upper and lower half surface of the coalescing bubble and their final convergence at its side, and (m)-(o) final detachment, upward jumping, rising, and oscillation with the Minnaert frequency. The images have been adopted from Ref. [165].

they are so large that the buoyant forces overwhelm the capillary forces (the so-called Fritz radius [75,164]) or when they coalesce and then detach, as shown in Fig. 23. In the context of catalysis and electrolysis, the bubble detachment is of course advantageous, as pointed out above.

The area of electrolysis and catalysis seems to me to be a very important and promising future field for physics of fluids, building a bridge from fluid dynamics to process technology and (colloid) chemistry.

\section{EFFECTIVE BUBBLE FORCE MODELS, DISPERSED BUBBLY FLOW, AND BUBBLE DRAG REDUCTION}

I now return from the predominantly diffusive bubble dynamics to further inertial bubble phenomena. As explained in Sec. II, the acoustic forces (Bjerknes forces) are the reason why the bubble is pushed towards the pressure antinode where it can oscillate in the strongest way, possibly even leading to single-bubble sonoluminescence [15]. In general, these acoustic forces will compete with inertial forces such as the added mass force, drag, and lift and of course with buoyancy. In 2001, we aimed to understand this competition in more detail in order to gain more insight into the hydrodynamic forces on bubbles such as drag and lift (which had very nicely been reviewed by Magnaudet and Eames [166]) and in order to better control the motion of bubbles in a shear flow with the help of an external acoustic field. We thus built an experimental setup with an acoustic spherical glass resonator through which we pumped some liquid in a controlled way, thus generating controlled shear flow [167]. Remarkably, for certain parameters, the entrapped bubbles performed a spiraling motion, out of which we could extract various pieces of information on the drag and in particular on the lift. The work, and follow-up work in similarly controlled geometries such as a rotating horizontal cylinder [168,169], also confirmed the usefulness of the concept of effective bubble forces, even for deformed or oscillating bubbles. In Fig. 24, for a bubble spiraling in the rotating horizontal cylinder [169], we show the forces acting on the bubble [Fig. 24(a)], the 

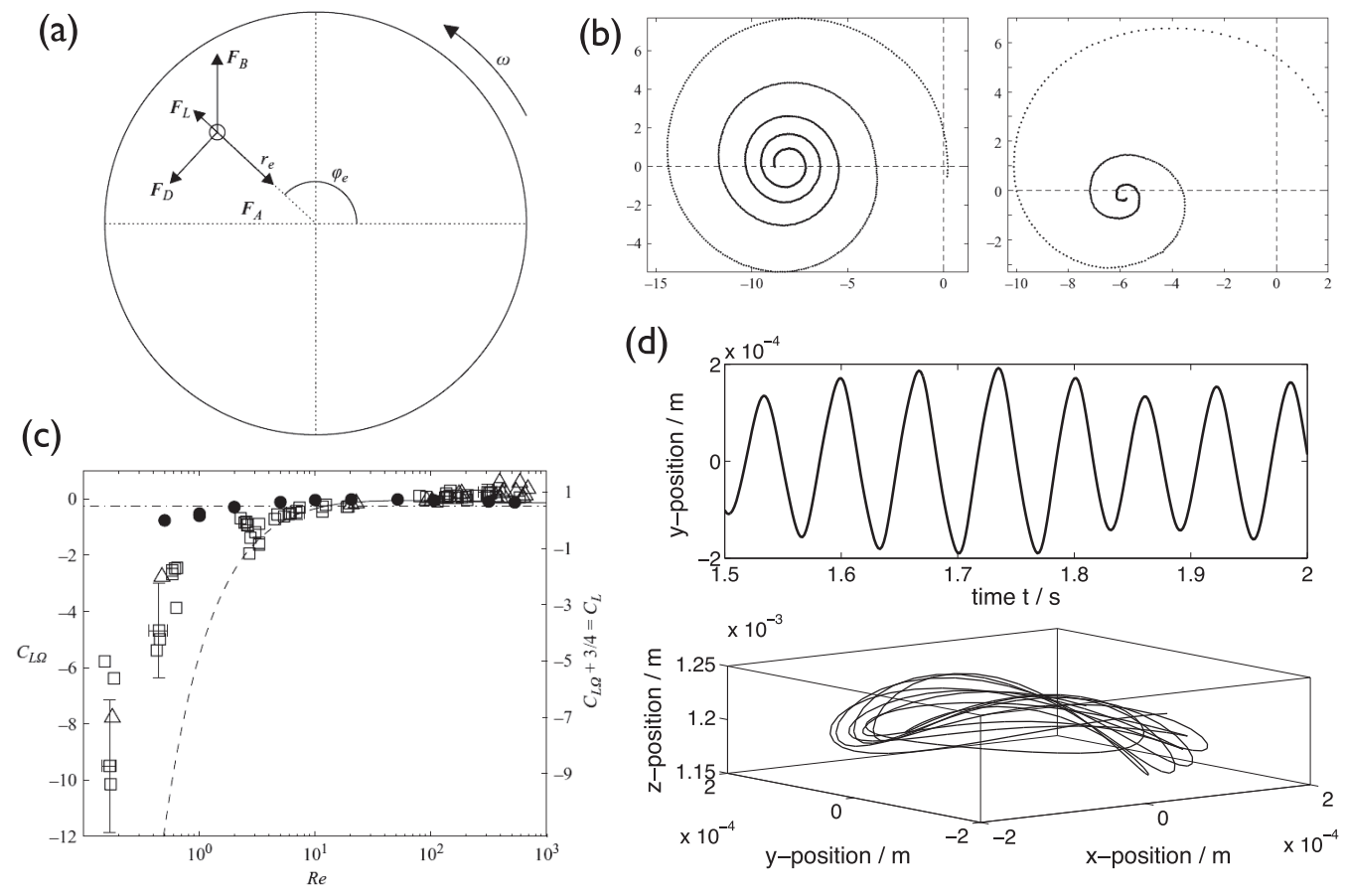

FIG. 24. (a) Forces acting on a bubble spiraling in a rotating horizontal cylinder: drag $F_{D}$, lift $F_{L}$, added mass $F_{A}$, and buoyancy $F_{B}$. The net force typically leads to a spiraling trajectory, as shown in (b) for bubbles with two different sets of parameters (axes in $\mathrm{mm}$ ); the center of the cylinder is at $(0,0)$. (c) Rotational lift coefficient $C_{L \Omega}$ extracted from such trajectories (open symbols) and compared with numerical data of Ref. [170]. (a)-(c) have been taken from Ref. [169], to which we refer for more details. (d) The $y$ position and three-dimensional trajectory of a moving SBSL bubble in $N$-methylformamide [172] from a dynamical model based on effective forces (including the history force), for the same parameters as in experiment [172], where similar trajectories are observed. The figures have been taken from Ref. [171].

spiraling trajectories for certain parameters [Fig. 24(b)], and the extracted rotational lift coefficients $C_{L}$, which remarkably can take both negative and positive values [Fig. 24(c)] [169]. In a particularly counterintuitive example, employing the Basset history force as a nonlocal-in-time effective force [170], we could explain how viscosity can destabilize the path dynamics of sonoluminescing bubbles [171], a phenomenon discovered by Suslick and co-workers for sonoluminescing bubbles in glycol and called moving single-bubble sonoluminescence (m-SBSL) [172]. The unstable, chaotic path trajectory of such a bubble is shown in Fig. 24(d).

Encouraged by these successes, we [173,174] dared to transfer Maxey and Riley's seminal idea of using effective forces on small particles to describe their (collective) dynamics in dispersed multiphase flow with pointlike particles [175] to turbulent dispersed multiphase flow [176-178] with pointlike bubbles, following Refs. [179-189]. The motion of the small and thus nondeformable bubbles of radius $a$, volume $\mathcal{V}_{b}=4 \pi a^{3} / 3$, velocity $\boldsymbol{v}(\boldsymbol{x}, t)$, and embedded in a velocity field $\mathbf{u}(\boldsymbol{x}, t)$ was modeled by (see, e.g., [166,179,186,187])

$$
\begin{aligned}
\rho_{g} \mathcal{V}_{b} \frac{d \boldsymbol{v}}{d t}= & \left(\rho_{g}-\rho_{f}\right) \mathcal{V}_{b} \boldsymbol{g}-C_{D} \frac{\pi a^{2}}{2} \rho_{f}|\boldsymbol{v}-\boldsymbol{u}|(\boldsymbol{v}-\boldsymbol{u}) \\
& +\rho_{f} \mathcal{V}_{b}\left(C_{M} \frac{D \boldsymbol{u}}{D t}-\frac{d \boldsymbol{v}}{d t}\right)+\rho_{f} \mathcal{V}_{b} \frac{D \boldsymbol{u}}{D t}-C_{L} \rho_{f} \mathcal{V}_{b}(\boldsymbol{v}-\boldsymbol{u}) \times \boldsymbol{\omega}
\end{aligned}
$$


(a)

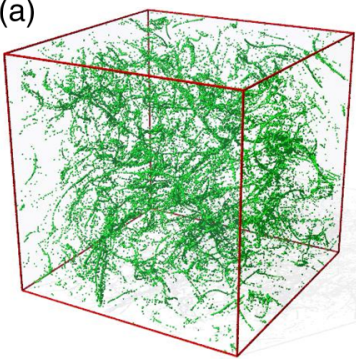

(b)

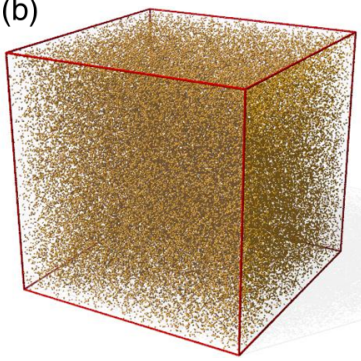

(c)

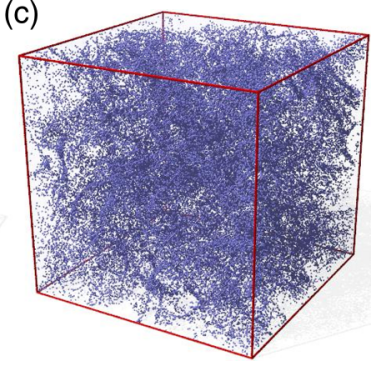

FIG. 25. Snapshots of the particle distribution in turbulent flow field for a Stokes number $\mathrm{St}=0.6$ for (a) bubbles, (b) tracers, and (c) heavy particles, all for a Taylor-Reynolds number $\operatorname{Re}_{\lambda}=75$, obtained with the point-particle approach in the spirit of Maxey and Riley [175]. The figure has been taken from Ref. [192].

and each bubble at position $\boldsymbol{y}(t)$ exerted a $\delta$-type force on the liquid flow [174],

$$
\boldsymbol{f}_{b}(\boldsymbol{x}, t)=\mathcal{V}_{b}\left(\frac{D \boldsymbol{u}}{D t}-\boldsymbol{g}\right) \delta(\boldsymbol{x}-\boldsymbol{y}(t)) \text {. }
$$

Here $\rho_{f}$ is the liquid density and $\rho_{g} \ll \rho_{f}$ is the (low) gas density. The terms on the right-hand side of Eq. (9) represent buoyancy, drag, added mass plus fluid acceleration, and lift, where $C_{D}$, $C_{M}$, and $C_{L}$ are the corresponding coefficients, which are modeled. Though in such complicated turbulent flow situation in particular the lift coefficient is not exactly known, as an approximation, and in order to explore the effect of the lift force on the overall flow dynamics, we took the standard value $C_{L}=1 / 2$ [166] for small spherical bubbles in shear flow. Following this approach, we studied the effect of the bubbles on the turbulent energy spectra [174], the Lagrangian statistics of bubbles in turbulence [190] finding very pronounced intermittency of the bubble acceleration, and the clustering of bubbles in the vortices [191,192] (see Fig. 25), in which we compare the distribution of bubbles with that of particles in dispersed multiphase flow. We also extended the effective bubble force idea to vapor bubbles in turbulent flow which can shrink and grow thanks to evaporation and condensation [193-195]. In this way we could reveal the physics of the considerable enhanced heat transfer in boiling thermal convection as compared to normal thermal convection. Two enhancing effects compete: enhancement due to additional mixing by the rising vapor bubbles and enhancement due to the bubbles as directed carriers of (latent) heat from the boiling bottom plate to the cold top plate. Due to the many modeling assumptions, a quantitative comparison with experimental data [117] however remains difficult.

One of the most remarkable features of dispersed bubbly flow is that a bubble addition of only a few percent leads to remarkable drag reduction of up to $80 \%$ [196,197], with great potential for naval applications [198] [see Fig. 26(a)], as it can lead to significant reduction of the fuel consumed by ships without adding substances into water. As far as I can remember, I myself first heard of this phenomenon at an APS DFD meeting in a talk of Kazu Sugiyama, then a postdoc in the group of Yoichiro Matsumoto and Shu Takagi in Tokyo, Japan. For excellent recent reviews on bubble drag reduction I refer to Refs. [200-202].

Despite all efforts done to understand the fundamental mechanisms behind this effect, a solid understanding of the drag reduction mechanisms occurring in bubbly flows is still missing. To achieve such an understanding, we wanted to study bubble drag reduction under the well-defined conditions of turbulent Taylor-Couette (TC) flow, the flow between two coaxial co- or counterrotating cylinders. For recent reviews on low-Reynolds-number and high-Reynolds-number TC flow, we refer to Refs. [203,204], respectively. The advantage of this canonical flow of physics of fluids is that it takes place in a closed system and that both global measurements (the overall torque required to drive the system, i.e., the drag) and local measurements (by particle image velocimetry or laser Doppler velocimetry) are possible. In a series of papers on turbulent bubble drag reduction [199,205,206], we could indeed measure major bubble drag reduction in this TC system, namely, in 
(a)

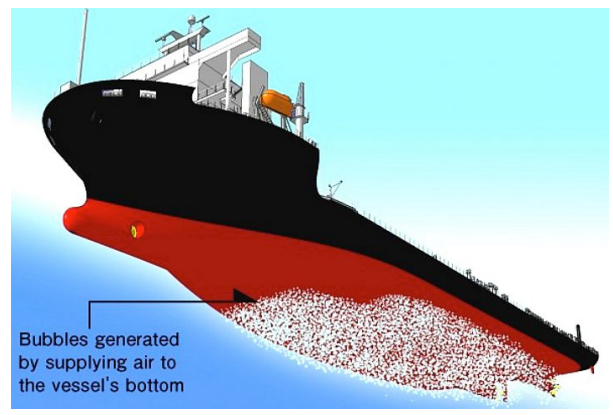

(b)

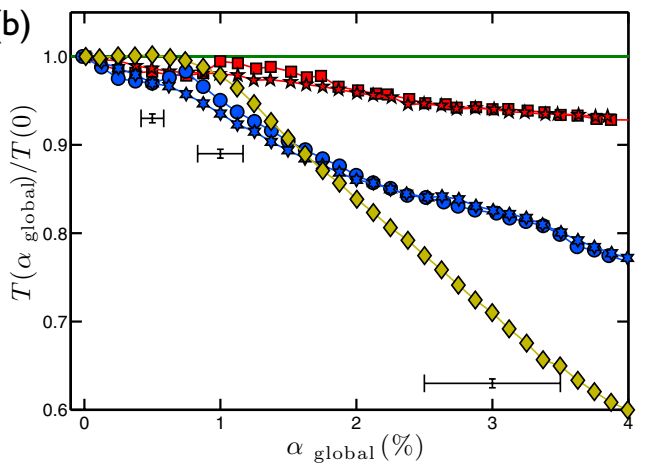

FIG. 26. (a) Sketch of the envisioned bubble drag reduction for naval transportation (Mitsubishi corporation, Japan). Bubbles are injected under the ship hull. (b) Drag reduction as a function of bubble volume concentration in turbulent bubbly Taylor-Couette flow for three different Reynolds numbers of the rotating inner cylinder, namely, $\operatorname{Re}=0.5 \times 10^{6}$ (red data), $\operatorname{Re}=1.0 \times 10^{6}$ (blue data), and $\operatorname{Re}=2.0 \times 10^{6}$ (yellow data). The vertical axis shows the required torque with bubbles divided by the required torque in the single-flow case. The figure has been taken from Ref. [199].

the strongly turbulent case up to $40 \%$ with only $4 \%$ volume fraction of the bubbles [see Fig. 26(b)] and in addition characterize bubble concentration and velocity profiles. The measurements were consistent with the conjecture that it is the bubble deformability that is responsible for the strong drag reduction, i.e., we only found substantial drag reduction for bubble Weber numbers We larger
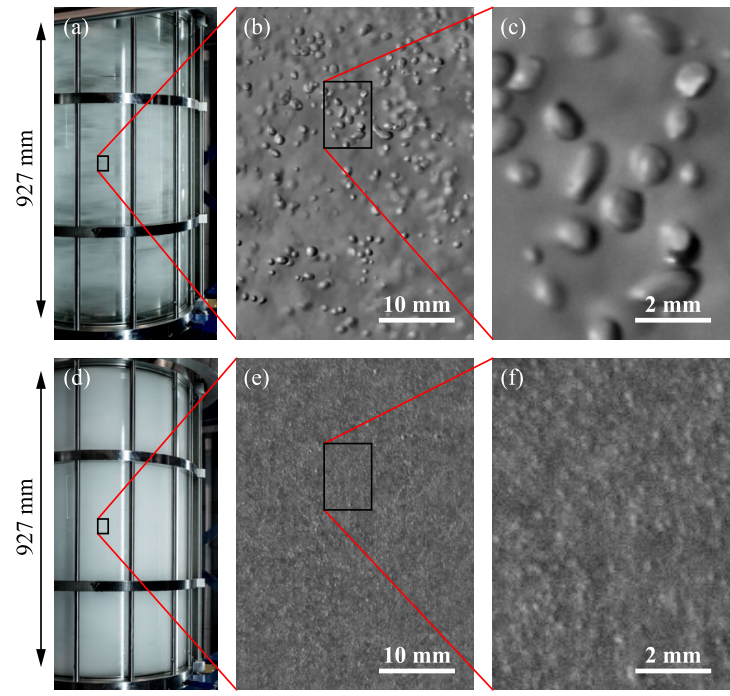

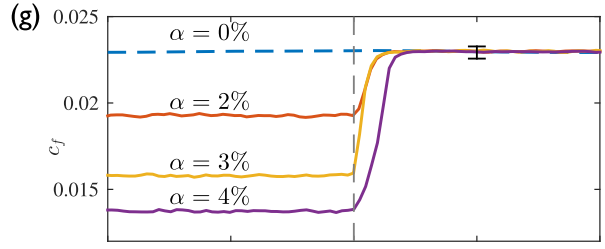

(h)

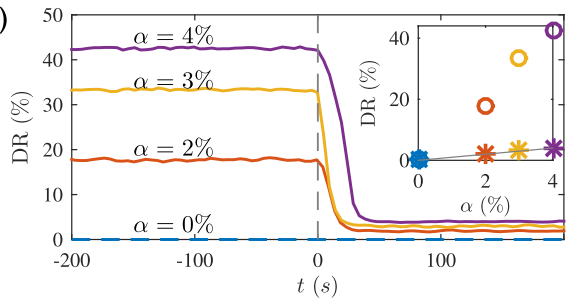

FIG. 27. (a)-(f) Snapshots of bubbly Taylor-Couette turbulence $\left(\alpha=1 \%, \operatorname{Re}_{i}=2 \times 10^{6}\right)$ with increasing magnification (as shown by the scale bars). In (a)-(c) no surfactants are present in the turbulent flow, whereas (d)-(f) show the (statistically stationary) situation after addition of 6 ppm Triton X-100. In (a) and (d) the Twente Turbulent Taylor-Couette $\left(\mathrm{T}^{3} \mathrm{C}\right)$ apparatus [210] can be seen. (g) Friction coefficient $c_{f}$ as a function of time for various bubble volume concentrations $\alpha$. The surfactant is added at time $t=0 \mathrm{~s}$. (h) Corresponding drag reduction (DR) (in \%) as a function of time. The inset shows how the drag reduction depends on the bubble volume concentrations: open circles, without surfactant; stars, with surfactant. 2The figures have been taken from Ref. [211]. 
(a)

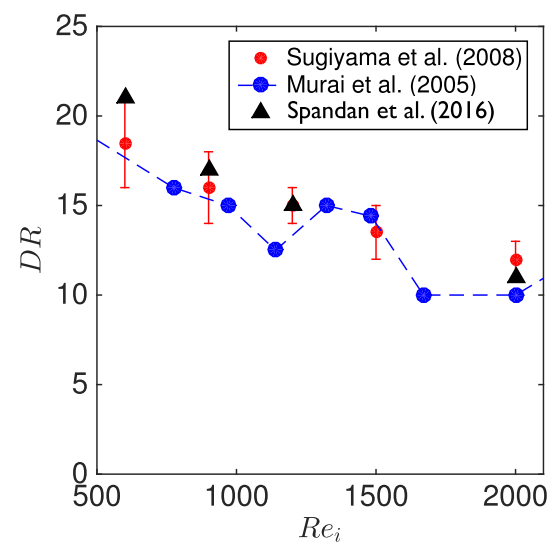

(b)

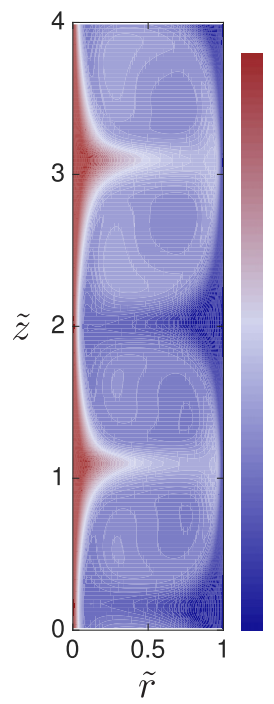

Single phase (c)

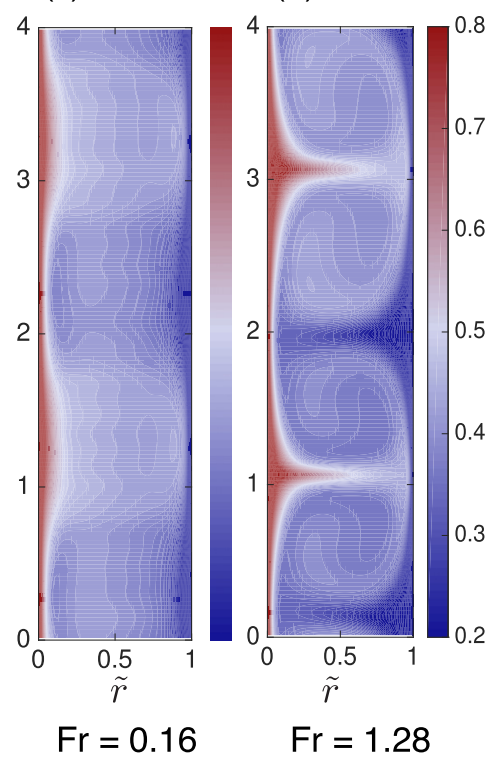

$\mathrm{Fr}=0.16$ (d)

FIG. 28. (a) Drag reduction DR as a function of the Reynolds number $\operatorname{Re}_{i}$ of the inner cylinder in TaylorCouette flow in the nonturbulent regime. The data are from the experiments by Murai et al. [214], the numerical simulations of Sugiyama et al. [215], and the numerical simulations by Spandan et al. [216]. One can clearly see that for these low Reynolds numbers the drag reduction is decreasing with increasing Reynolds numbers. (b)-(d) Corresponding averaged azimuthal velocity contours with point bubbles included. Here $\mathrm{Re}_{i}=2500$ and single-phase flow is compared with two different Froude numbers $\mathrm{Fr}=0.16$ (strong buoyancy, weakening the Taylor rolls considerable) and $\mathrm{Fr}=1.28$ (weak buoyancy). In the latter case, the Taylor rolls are hardly affected by the bubbles which get trapped in them. The figures have been taken from Ref. [216].

than 1 [199]. These findings were in line with the results from numerical simulations by Gretar Tryggvason's group [207], employing the front-tracking technique. The modification of the lift force on the bubbles due to their deformability (for We $>1$ ) seems to play a central role in the mechanism and the bubbles arrange themselves in between the turbulent bulk and the boundary layers, blocking the momentum transfer and therefore reducing the overall drag.

How is this hypothesis further validated? If the hypothesis were correct, one should change the bubble deformability during the experiment and then the drag should change on the spot. How can this be achieved? In 2010 I had visited the group of Yoichiro Matsumoto and Shu Takagi in Tokyo and saw their impressive huge bubble column experiment, in which the addition of a few drops of surfactants (Triton X) immediately changed the flow characteristics and bubble size (see Ref. [208]); for a recent review of this phenomenon, see [209]. The main reason is that the Triton X surfactant prevents bubble coalescence and therefore, in strongly turbulent flow in which bubble splitting is omnipresent, the average bubble size will dramatically decrease after the addition of Triton X.

We decided to apply the same trick in turbulent bubbly TC flow and indeed found that also here the addition of a few drops of Triton X (to 111 liter of water in our TC setup [210]) dramatically changed the flow [211] (see Fig. 27) in spite of the unchanged bubble volume fraction: optically [Figs. 27(a)-27(f)], because the flow became very opaque, just as in Ref. [208], but in particular also mechanically [Figs. 27(g) and 27(h)], as the effect of bubble drag reduction nearly vanished. The combination of these local and global findings strongly supports the hypothesis that it is the bubble deformability which is relevant for strong turbulent bubble drag reduction.

How is turbulent bubble drag reduction modeled numerically? Given that the mechanism is bubble deformability, which requires one to resolve the gas-liquid interfaces of the bubbles and 

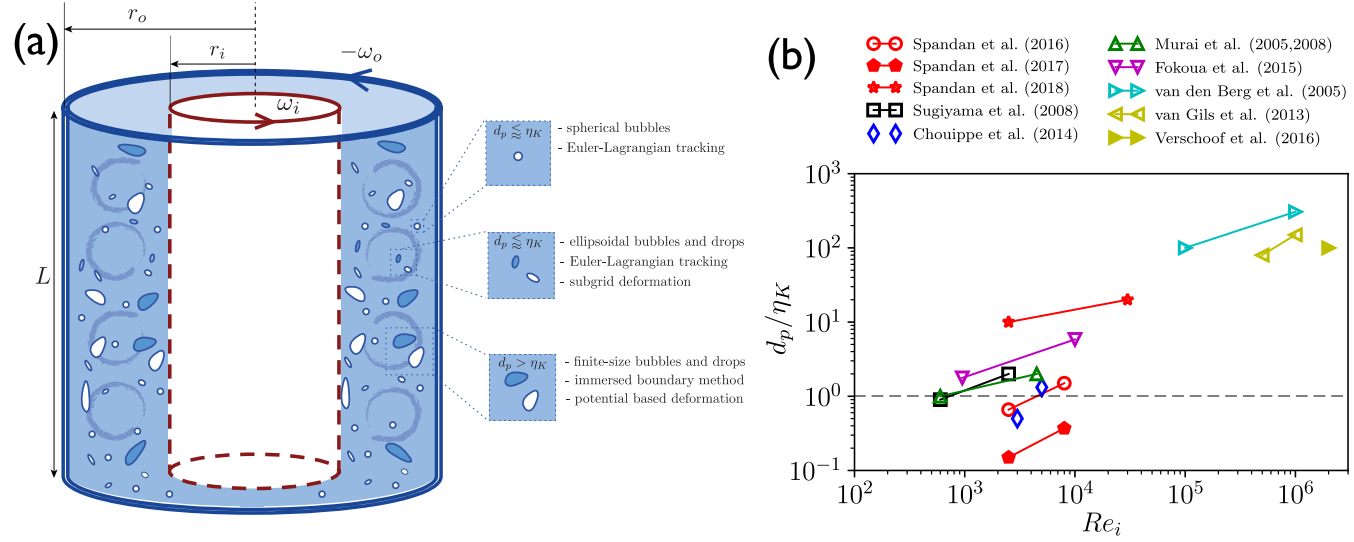

FIG. 29. (a) Visualization of the different ways to numerically treat bubbles in TC flow, depending on their sizes. The figure has been adopted from the Ph.D. thesis of Vamsi Spandan, Physics of Fluids, University of Twente, 2017. (b) Parameter space of bubble drag reduction in TC flow: Various bubble sizes $d_{b}$ (given relative to the Kolmogorov scale $\delta_{v}$ ) were realized in experiments and simulations for Reynolds numbers $\operatorname{Re}_{i}$ from a few hundred up to more than $10^{6}$. The left block of data is experiments and numerical simulations for microbubble drag reduction from Refs. [214,215,218,219] and the right block of data is our large Re experiments with deformable bubbles $[199,205,206]$.

given the large Reynolds numbers, this is extremely challenging. With the front-tracking technique, Tryggvason and co-workers could simulate a few large bubbles in a minimum turbulent channel, indeed qualitatively finding bubble drag reduction due to deformability [207]. Also his later work with more bubbles confirmed this interpretation [212,213].

For the numerical simulations of bubble drag reduction in TC flow, we started off moderately, namely, for relatively small Reynolds numbers (based on the inner cylinder rotation; the outer cylinder was at rest) $\mathrm{Re}_{i}<5000$, for which the flow is not yet turbulent. Note that these Reynolds numbers are more than two orders of magnitude smaller than what I discussed in Figs. 26 and 27. Interestingly enough, also in that low-Re regime Murai et al. [214] had experimentally found bubble drag reduction, namely, by microbubble injection [see Fig. 28(a)]. However, due to the lack of bubble deformability (We $<1$ for the injected microbubbles), the physical mechanism in that regime must be very different. The good news is that this regime is accessible to numerical simulations, and even with effective force models, as the microbubbles can be considered as pointlike, with We $<1$ and a diameter $d_{b}<10 \eta$, where $\eta$ is the (global) Kolmogorov length of the flow.

Our numerical simulations of bubbly TC flow at these low $\mathrm{Re}_{i}<5000$ and with pointlike bubbles [215,216] indeed resulted in bubble drag reduction in quantitative agreement with Murai's data [214] [see Fig. 28(a)]. In contrast to the bubble drag reduction in the large-Reynolds-number regime, which increases with increasing Reynolds number [Fig. 26(b)], in the low-Reynolds-number regime the effect of bubble drag reduction gets smaller with increasing Reynolds number [Fig. 28(a)].

As the relevant mechanism for bubble drag reduction in TC flow in the low-Reynolds-number regime, we could identify the considerable weakening of the Taylor rolls through the rising bubbles [see Figs. 28(b)-28(d)]. The crucial parameter in this low-Reynolds-number regime is therefore the Froude number (and not the Weber number as in the large Reynolds-number regime), here defined as the square root of the ratio between centrifugal force and buoyancy, $\mathrm{Fr}=\omega_{i} \sqrt{r_{i} / g}$, where $r_{i}$ is the bubble radius, $\omega_{i}$ the angular velocity of the inner cylinder, and $g$ gravity. For small $\mathrm{Fr}<1$ [Fig. 28(c)], buoyancy wins and the bubbles rise through the Taylor rolls, thereby weakening them. This reduces the angular momentum transfer from the inner to the outer cylinder and thus the drag. For large Fr $>1$ [Fig. 28(d)] the bubbles simply get trapped in the Taylor rolls, without affecting them much, and the overall drag remains nearly unchanged [217]. For increasing Reynolds numbers 
(a)

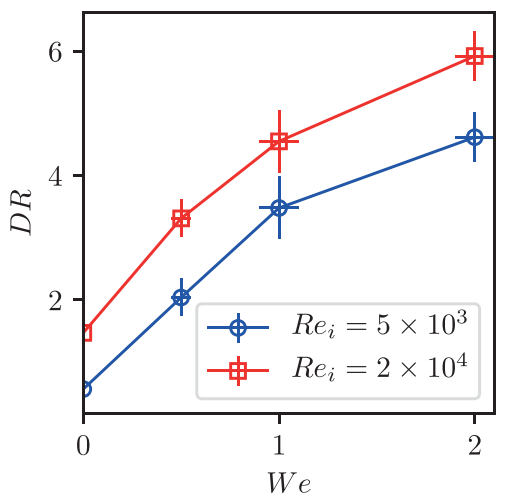

(b)
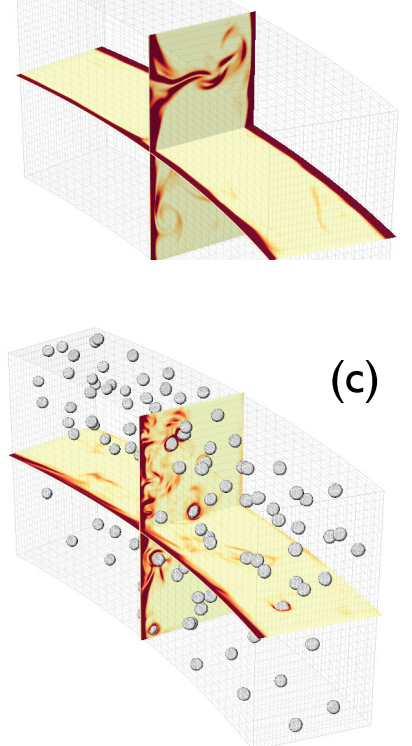

FIG. 30. (a) Drag reduction in turbulent TC flow as a function of Weber number for two different Reynolds numbers. Increasing Weber number implies increasing bubble deformability, which is crucial for the drag reduction. The figure has been taken from the numerical simulations of Ref. [222]. Also shown are numerical simulations showing plume detachment from the inner TC cylinder for (b) single-phase flow and (c) bubbly flow, where the momentum transfer is considerably depressed. In the latter case deformable bubbles considerably lower the momentum transfer. The calculation was done with the immersed boundary method.

the Taylor rolls more and more lose their flow dominance, which explains why in this low-Reynoldsnumber regime the bubble drag reduction decreases with increasing Reynolds number [Fig. 28(a)].

The challenge for the next years will be to close the gap between the microbubble drag reduction experiments at lower Re of Murai et al. [214] and our large-Re experiments with deformable bubbles [199] (see Fig. 29). Our first step toward this was to include bubble deformability in point particles by picking up an idea of Refs. [220,221] and couple the deformation dynamics of ellipsoidal bubbles to the flow. Indeed, we found that bubble deformation helped the overall drag reduction [217]. In the second step [222], we coupled the well-resolved bubbles with an immersed boundary method to the Navier-Stokes equation, again finding that an increase in the bubble deformability (i.e., its Weber number) indeed implies larger drag reduction [see Fig. 30(a)]. Profiles of the local angular velocity flux show that, in the presence of bubbles, turbulence is enhanced near the inner cylinder while attenuated in the bulk and near the outer cylinder. We connect the increase in drag reduction to the decrease in dissipation in the wake of highly deformed bubbles near the inner cylinder [222], as visualized in Figs. 30(b) and 30(c). As in the closed TC system the total dissipation is proportional to the total torque [204], the decrease in dissipation implies a decrease in the overall drag.

Bringing all these fundamental insights into bubble drag reduction towards applications in naval industry, however, still is a very long way to go and other concepts like air cavities may be more efficient [223]. We are presently collaborating with the Dutch Maritime Research Institute (MARIN) in Wageningen on this subject.

\section{CONCLUSION}

I hope to have demonstrated, along my own bubble trajectory, how fascinating and broad the fluid dynamics of a bubble is, from the nanometer scale to geophysical scales, with both outstanding 
fundamental questions and relevant applications. Clearly, the close interaction between experiment, theory, and numerics is crucial in the physics of fluids and in particular in the physics of bubbles and makes this subject particularly nice. Only a combination of all three methods gives real insight into the phenomena.

I also hope to have demonstrated with the chosen examples how crucial and essential for innovation of any kind fundamental research is. In what direction creative fundamental research will go is often unpredictable. If one ends up exactly where one had expected, the chance is large that the research was standard. What remains crucial is to be open, watch, and listen.

I want to close with some more general comments on fluid dynamics. From my point of view, we presently live in the golden age of fluid dynamics. The reasons are that (i) Moore's law is still being followed for the computational power, now making simulations possible, which even ten years ago we did not dare to dream of, and (ii) there has been a similar revolution (for the same reason) in digital high-speed imaging, now being able to routinely resolve the millisecond timescale and even smaller timescales [224], revealing new physics on these scales which up to now was inaccessible and producing a huge amount of data on the flow. Also other advanced equipment like confocal microscopy, digital holographic microscopy, and atomic force microscopy get more and more used in fluid dynamics. With all of these advances together, the gap between what can be measured and what can be ab initio simulated is more quickly closing than we had anticipated at the end of the past century.

Also other gaps are closing: Fluid dynamics and in particular bubble fluid dynamics are bridging various neighboring disciplines such as chemistry and in particular colloidal science, catalysis, electrolysis, medicine, biology, computational science, and many others. Here the techniques, approaches, and traditions from fluid dynamics can offer a lot to help to solve outstanding problems and vice versa, these fields can offer wonderful questions to fluid dynamics. Academic (bubble) fluid dynamics is also bridging out not only to traditional applications on large scales such as in chemical engineering, in the food industry, or in geophysics, but also to various new high-tech applications, be it in inkjet printing, immersion and XUV lithography, chemical diagnostics, and laboratory-on-a-chip microfluidics.

\section{ACKNOWLEDGMENTS}

Science is a collective activity. The scientific insight in this paper is gained during my bubble journey over more than two decades together with my colleagues, postdocs, Ph.D. students, and students and I would like to thank them for all their work and contributions, and for the stimulation and intellectual pleasure we have enjoyed when doing physics. But first of all, I would like to express my sincere gratitude to my scientific teacher, Professor Siegfried Grossmann, for all what I learnt from him, which goes way beyond physics. Then, in particular, on this subject of bubbles, I would like to thank Michael Brenner, Sascha Hilgenfeldt and Michel Versluis, with whom my bubbly journey started, and the "part-time professors" of our Twente Physics of Fluids group, namely my colleagues and friends Andrea Prosperetti (who also inspired us on the beauty of bubbles in art [225]!), Roberto Verzicco, Chao Sun, and Xuehua Zhang, whose intellectual contribution to our group is way beyond "part-time", from whom I learned tremendously, and with whom it has been a great pleasure and privilege to work. I also gratefully acknowledge generous financial support over the years, mainly from NWO via various programs and from the European Union via ERC Advanced Grants.

[1] C. E. Brennen, Cavitation and Bubble Dynamics (Oxford University Press, Oxford, 1995).

[2] T. G. Leighton, The Acoustic Bubble (Academic, London, 1994).

[3] D. Lohse, Bubble puzzles, Nonlinear Phenom. Complex Syst. 9, 125 (2006).

[4] B. P. Barber, Synchronous picosecond sonoluminescence, Ph.D. thesis, University of California at Los Angeles, 1992. 
[5] B. P. Barber and S. J. Putterman, Observation of synchronous picosecond sonoluminescence, Nature (London) 352, 318 (1991).

[6] B. P. Barber, R. A. Hiller, R. Löfstedt, S. J. Putterman, and K. R. Weninger, Defining the unknowns of sonoluminescence, Phys. Rep. 281, 65 (1997).

[7] D. F. Gaitan, An experimental investigation of acoustic cavitation in gaseous liquids, Ph.D. thesis, University of Mississippi, 1990.

[8] L. A. Crum, Sonoluminescence, Phys. Today 47(9), 22 (1994).

[9] V. Bjerknes, Die Kraftfelder (Vieweg, Braunschweig, 1909).

[10] A. Prosperetti, Current topics in the dynamics of gas and vapor bubbles, Meccanica 12, 214 (1977).

[11] D. F. Gaitan, L. A. Crum, C. C. Church, and R. A. Roy, Sonoluminescence and bubble dynamics for a single, stable, cavitation bubble, J. Acoust. Soc. Am. 91, 3166 (1992).

[12] M. S. Plesset and A. Prosperetti, Bubble dynamics and cavitation, Annu. Rev. Fluid Mech. 9, 145 (1977).

[13] L. Rayleigh, On the pressure developed in a liquid during the collapse of a spherical cavity, Philos. Mag. 34, 94 (1917).

[14] D. Lohse, Bubble puzzles, Phys. Today 56(2), 36 (2003).

[15] M. P. Brenner, S. Hilgenfeldt, and D. Lohse, Single bubble sonoluminescence, Rev. Mod. Phys. 74, 425 (2002).

[16] A. Eller and L. A. Crum, Instability of motion of a pulsating bubble in a sound field, J. Acoust. Soc. Am. Suppl. 47, 762 (1970).

[17] A. Prosperetti, Viscous effects on perturbed spherical flows, Q. Appl. Math. 34, 339 (1977).

[18] M. P. Brenner, D. Lohse, and T. F. Dupont, Bubble Shape Oscillations and the Onset of Sonoluminescence, Phys. Rev. Lett. 75, 954 (1995).

[19] S. Hilgenfeldt, D. Lohse, and M. P. Brenner, Phase diagrams for sonoluminescing bubbles, Phys. Fluids 8, 2808 (1996).

[20] P. S. Epstein and M. S. Plesset, On the stability of gas bubbles in liquid-gas solutions, J. Chem. Phys. 18, 1505 (1950).

[21] M. M. Fyrillas and A. J. Szeri, Dissolution or growth of soluble spherical oscillating bubbles, J. Fluid Mech. 277, 381 (1994).

[22] F. G. Blake, Bjerknes forces in stationary sound fields, J. Acoust. Soc. Am. 21, 551 (1949).

[23] A. Eller and H. G. Flynn, Rectified Diffusion During Nonlinear Pulsations of Cavitation Bubbles, J. Acoust. Soc. Am. 37, 493 (1964).

[24] L. A. Crum, Measurements of the growth of air bubbles by rectified diffusion, J. Acoust. Soc. Am. 68, 203 (1980).

[25] M. P. Brenner, D. Lohse, D. Oxtoby, and T. F. Dupont, Mechanisms for Stable Single Bubble Sonoluminescence, Phys. Rev. Lett. 76, 1158 (1996).

[26] D. Lohse, M. P. Brenner, T. F. Dupont, S. Hilgenfeldt, and B. Johnston, Sonoluminescing Air Bubbles Rectify Argon, Phys. Rev. Lett. 78, 1359 (1997).

[27] R. Toegel and D. Lohse, Phase diagrams for sonoluminescing bubbles: A comparison between experiment and theory, J. Chem. Phys. 118, 1863 (2003).

[28] D. J. Flannigan and K. S. Suslick, Plasma formation and temperature measurement during single-bubble cavitation, Nature (London) 434, 52 (2005).

[29] T. J. Matula and L. A. Crum, Evidence for Gas Exchange in Single-Bubble Sonoluminescence, Phys. Rev. Lett. 80, 865 (1998).

[30] J. A. Ketterling and R. E. Apfel, Experimental Validation of the Dissociation Hypothesis for Single Bubble Sonoluminescence, Phys. Rev. Lett. 81, 4991 (1998).

[31] W. C. Moss, D. B. Clarke, and D. A. Young, Calculated pulse widths and spectra of a single sonoluminescing bubble, Science 276, 1398 (1997).

[32] S. Hilgenfeldt, S. Grossmann, and D. Lohse, A simple explanation of light emission in sonoluminescence, Nature (London) 398, 402 (1999).

[33] P. Frinking, A. Bouakaz, J. Kirkhorn, F. Ten Cate, and N. de Jong, Ultrasound contrast imaging: Current and new potential methods, Ultrasound Med. Biol. 26, 965 (2000). 
[34] H. Becher and P. N. Burns, Handbook of Contrast Echocardiography: Left Ventricular Function and Myocardial Perfusion (Springer, New York, 2000).

[35] S. R. Wilson and P. N. Burns, Microbubble-enhanced US in Body Imaging: What Role? Radiology 257, 24 (2010).

[36] F. Cavalieri, M. Ashokkumar, F. Grieser, and F. Caruso, Ultrasonic synthesis of stable, functional lysozyme microbubbles, Langmuir 24, 10078 (2008).

[37] J. S. Lum, J. D. Dove, T. W. Murray, and M. A. Borden, Single microbubble measurements of lipid monolayer viscoelastic properties for small-amplitude oscillations, Langmuir 32, 9410 (2016).

[38] P. Marmottant, S. van der Meer, M. Versluis, N. de Jong, S. Hilgenfeldt, and D. Lohse, A model for large amplitude oscillations of coated bubbles accounting for buckling and rupture, J. Acoust. Soc. Am. 118, 3499 (2005).

[39] B. Dollet, P. Marmottant, and V. Garbin, Bubble dynamics in soft and biological matter, Annu. Rev. Fluid. Mech. 51, 331 (2019).

[40] P. N. Burns, Harmonic imaging with ultrasound contrast agents, Clin. Radiol. 51, 50 (1996).

[41] A. Prosperetti, Bubble phenomena in sound fields, part one, Ultrasonics 22, 115 (1984).

[42] S. Grossmann, S. Hilgenfeldt, D. Lohse, and M. Zomack, Sound radiation of 3-MHz driven gas bubbles, J. Acoust. Soc. Am. 102, 1223 (1997).

[43] C. T. Chin, C. Lancee, J. Borsboom, F. Mastik, M. E. F. N. de Jong, M. Versluis, and D. Lohse, Brandaris 128: A digital 25 million frames per second camera with 128 highly sensitive frames, Rev. Sci. Instrum. 74, 5026 (2003).

[44] E. C. Gelderblom, H. J. Vos, F. Mastik, T. Faez, Y. Luan, T. J. A. Kokhuis, A. F. W. van der Steen, D. Lohse, N. de Jong, and M. Versluis, Brandaris 128 ultra-high-speed imaging facility: 10 years of operation, updates, and enhanced features, Rev. Sci. Instrum. 83, 103706 (2012).

[45] S. M. van der Meer, B. Dollet, M. M. Voormolen, C. T. Chin, A. Bouakaz, N. de Jong, M. Versluis, and D. Lohse, Microbubble spectroscopy of ultrasound contrast agents, J. Acoust. Soc. Am. 121, 648 (2007).

[46] B. Dollet, S. M. van der Meer, V. Garbin, N. de Jong, D. Lohse, and M. Versluis, Nonspherical oscillations of ultrasound contrast agent microbubbles, Ultrasound Med. Biol. 34, 1465 (2008).

[47] M. Overvelde, V. Garbin, J. Sijl, B. Dollet, N. de Jong, D. Lohse, and M. Versluis, Nonlinear shell behavior of phosolipid-coated microbubbles, Ultrasound Med. Biol. 36, 2080 (2010).

[48] N. De Jong, M. Emmer, C. T. Chin, A. Bouakaz, F. Mastik, D. Lohse, and M. Versluis, "Compressiononly" behavior of phospholipid-coated contrast bubbles, Ultrasound Med. Biol. 33, 653 (2007).

[49] J. Sijl, M. Overvelde, B. Dollet, V. Garbin, N. de Jong, D. Lohse, and M. Versluis, Compression-only behavior: A second-order nonlinear response of ultrasound contrast agent microbubbles, J. Acoust. Soc. Am. 129, 1729 (2011).

[50] S. L. Anna, N. Bontoux, and H. A. Stone, Formation of dispersions using "flow focusing" in microchannels, Appl. Phys. Lett. 82, 364 (2003).

[51] A. Utada, E. Lorenceau, D. Link, P. Kaplan, H. Stone, and D. Weitz, Monodisperse double emulsions generated from a microcapillary device, Science 308, 537 (2005).

[52] A. S. Utada, A. Fernandez-Nieves, H. A. Stone, and D. A. Weitz, Dripping to Jetting Transitions in Coflowing Liquid Streams, Phys. Rev. Lett. 99, 094502 (2007).

[53] J. M. Gordillo, Z. Cheng, A. M. Ganan-Calvo, M. Marquez, and D. A. Weitz, A new device for the generation of microbubbles, Phys. Fluids 16, 2828 (2004).

[54] E. Castro-Hernandez, W. van Hoeve, D. Lohse, and J. M. Gordillo, Microbubble generation in a co-flow device operated in a new regime, Lab Chip 11, 2023 (2011).

[55] C. D. Ohl, M. Arora, R. Ikink, N. D. Jong, M. Versluis, M. Delius, and D. Lohse, Sonoporation from jetting cavitation bubbles, Biophys. J. 91, 4285 (2006).

[56] O. Shpak, M. Verweij, H. J. Vos, N. de Jong, D. Lohse, and M. Versluis, Acoustic droplet vaporization is initiated by superharmonic focusing, Proc. Natl. Acad. Sci. USA 111, 1697 (2014).

[57] A. Prosperetti, Vapor bubbles, Annu. Rev. Fluid Mech. 49, 221 (2017).

[58] Y. Wang, M. E. Zaytsev, H. L. The, J. C. T. Eijkel, H. J. W. Zandvliet, X. Zhang, and D. Lohse, Vapor and gas-bubble growth dynamics around laser-irradiated, water-immersed plasmonic nanoparticles, ACS Nano 11, 2045 (2017). 
[59] Z. Fang, Y. R. Zhen, O. Neumann, A. Polman, F. J. Garcia de Abajo, P. Nordlander, and N. J. Halas, Evolution of light-induced vapor generation at a liquid-immersed metallic nanoparticle, Nano Lett. 13, 1736 (2013).

[60] G. Baffou, J. Polleux, H. Rigneault, and S. Monneret, Super-heating and micro-bubble generation around plasmonic nanoparticles under cw illumination, J. Phys. Chem. C 118, 4890 (2014).

[61] D. Lapotko, Plasmonic nanoparticle-generated photothermal bubbles and their biomedical applications, Nanomedicine 4, 813 (2009).

[62] S. Y. Emelianov, P.-C. Li, and M. O'Donnell, Photoacoustics for molecular imaging and therapy, Phys. Today 62(5), 34 (2009).

[63] G. Baffou and R. Quidant, Thermo-plasmonics: Using metallic nanostructures as nano-sources of heat, Laser Photon. Rev. 7, 171 (2013).

[64] J. Shao, M. Xuan, L. Dai, T. Si, J. Li, and Q. He, Near-infrared-activated nanocalorifiers in microcapsules: Vapor bubble generation for in vivo enhanced cancer therapy, Angew. Chem. Int. Ed. 54, 12782 (2015).

[65] H.-L. Liu, C.-H. Fan, C.-Y. Ting, and C.-K. Yeh, Combining microbubbles and ultrasound for drug delivery to brain tumors: Current progress and overview, Theranostics 4, 432 (2014).

[66] O. Neumann, C. Feronti, A. D. Neumann, A. Dong, K. Schell, B. Lu, E. Kim, M. Quinn, S. Thompson, N. Grady, P. Nordlander, M. Oden, and N. J. Halas, Compact solar autoclave based on steam generation using broadband light-harvesting nanoparticles, Proc. Natl. Acad. Sci. USA 110, 11677 (2013).

[67] Y. Wang, M. E. Zaytsev, G. Lajoinie, H. L. The, J. C. T. Eijkel, A. van den Berg, B. M. Weckhuysen, X. Zhang, H. J. W. Zandvliet, and D. Lohse, Giant and explosive plasmonic bubbles by delayed nucleation, Proc. Natl. Acad. Sci. USA 115, 7676 (2018).

[68] A. Prosperetti, L. A. Crum, and H. C. Pumphrey, Underwater noise of rain, J. Geophys. Res. 94, 3255 (1989).

[69] M. Versluis, A. von der Heydt, B. Schmitz, and D. Lohse, How snapping shrimp snap: Through cavitating bubbles, Science 289, 2114 (2000).

[70] F. A. Everest, R. W. Young, and M. W. Johnson, Acoustical characteristics of noise produced by snapping shrimp, J. Acoust. Soc. Am. 20, 137 (1948).

[71] D. Lohse, B. Schmitz, and M. Versluis, Snapping shrimp make flashing bubbles, Nature (London) 413, 477 (2001).

[72] A. Prosperetti and H. Oguz, The impact of drops on liquid surfaces and the underwater noise of rain, Annu. Rev. Fluid Mech. 25, 577 (1993).

[73] H. Oguz and A. Prosperetti, Bubble entrainment by the impact of drops on liquid surfaces, J. Fluid Mech. 219, 143 (1990).

[74] H. N. Oguz, A. Prosperetti, and A. R. Kolaini, Air entrapment by a falling water mass, J. Fluid Mech. 294, 181 (1995).

[75] H. N. Oguz and A. Prosperetti, Dynamics of bubble-growth and detachment from a needle, J. Fluid Mech. 257, 111 (1993).

[76] D. Lohse, R. Bergmann, R. Mikkelsen, C. Zeilstra, D. van der Meer, M. Versluis, K. van der Weele, M. van der Hoef, and H. Kuipers, Impact on Soft Sand: Void Collapse and Jet Formation, Phys. Rev. Lett. 93, 198003 (2004).

[77] J. C. Burton, R. Waldrep, and P. Taborek, Scaling and Instabilities in Bubble Pinch-Off, Phys. Rev. Lett. 94, 184502 (2005).

[78] J. M. Gordillo, A. Sevilla, J. Rodriguez-Rodriguez, and C. Martinez-Bazan, Axisymmetric Bubble Pinch-Off at High Reynolds Numbers, Phys. Rev. Lett. 95, 194501 (2005).

[79] R. Bergmann, M. Stijnman, M. Sandtke, D. van der Meer, A. Prosperetti, and D. Lohse, Giant Bubble Collapse, Phys. Rev. Lett. 96, 154505 (2006).

[80] S. T. Thoroddsen, T. G. Etoh, and K. Takehara, Experiments on bubble pinch-off, Phys. Fluids 19, 042101 (2007).

[81] N. C. Keim, P. Møller, W. W. Zhang, and S. R. Nagel, Breakup of Air Bubbles in Water: Memory and Breakdown of Cylindrical Symmetry, Phys. Rev. Lett. 97, 144503 (2006). 
[82] R. P. H. M. Bergmann, D. van der Meer, S. Gekle, J. van der Bos, and D. Lohse, Controlled impact of a disk on a water surface: Cavity dynamics, J. Fluid Mech. 633, 381 (2009).

[83] J. M. Gordillo and M. Perez-Saborid, Axisymmetric breakup of bubbles at high Reynolds numbers, J. Fluid Mech. 562, 303 (2006).

[84] J. Eggers, M. A. Fontelos, D. Leppinen, and J. H. Snoeijer, Theory of the Collapsing Axisymmetric Cavity, Phys. Rev. Lett. 98, 094502 (2007).

[85] S. Gekle, J. Manuel Gordillo, D. van der Meer, and D. Lohse, High-Speed Jet Formation after Solid Object Impact, Phys. Rev. Lett. 102, 034502 (2009).

[86] O. R. Enriquez, I. R. Peters, S. Gekle, L. E. Schmidt, D. Lohse, and D. van der Meer, Collapse and pinch-off of a non-axisymmetric impact-created air cavity in water, J. Fluid Mech. 701, 40 (2012).

[87] S. Gekle, I. R. Peters, J. M. Gordillo, D. van der Meer, and D. Lohse, Supersonic Air Flow due to Solid-Liquid Impact, Phys. Rev. Lett. 104, 024501 (2010).

[88] A. Prosperetti, On the stability of spherically symmetric flows, Atti Accad. Naz. Lincei Rend. Cl. Sci. Fis. Mat. Nat. 62, 196 (1977).

[89] G. A. Caballero, R. Bergmann, D. van der Meer, A. Prosperetti, and D. Lohse, Role of Air in Granular Jet Formation, Phys. Rev. Lett. 99, 018001 (2007).

[90] J. R. Royer, E. I. Corwin, P. J. Eng, and H. M. Jaeger, Gas-Mediated Impact Dynamics in Fine-Grained Granular Materials, Phys. Rev. Lett. 99, 038003 (2007).

[91] T. Homan, C. Gjaltema, and D. van der Meer, Collapsing granular beds: The role of interstitial air, Phys. Rev. E 89, 052204 (2014).

[92] D. van der Meer, Impact on granular beds, Annu. Rev. Fluid Mech. 49, 463 (2017).

[93] J. de Jong, G. de Bruin, H. Reinten, M. van den Berg, H. Wijshoff, M. Versluis, and D. Lohse, Air entrapment in piezo-driven inkjet printheads, J. Acoust. Soc. Am. 120, 1257 (2006).

[94] R. J. M. Jeurissen, A. van der Bos, H. Reinten, M. van den Berg, H. Wijshoff, J. de Jong, M. Versluis, and D. Lohse, Acoustic measurement of bubble size in an inkjet printhead, J. Acoust. Soc. Am. 126, 2184 (2009).

[95] R. J. M. Jeurissen, J. de Jong, H. Reinten, M. van den Berg, H. Wijshoff, M. Versluis, and D. Lohse, Effect of an entrained air bubble on the acoustics of an ink channel, J. Acoust. Soc. Am. 123, 2496 (2008).

[96] J. de Jong, R. Jeurissen, H. Borel, M. van den Berg, H. Wijshoff, H. Reinten, M. Versluis, A. Prosperetti, and D. Lohse, Entrapped air bubbles in piezo-driven inkjet printing: Their effect on the droplet velocity, Phys. Fluids 18, 121511 (2006).

[97] W. Bouwhuis, R. C. A. van der Veen, T. Tran, D. L. Keij, K. G. Winkels, I. R. Peters, D. van der Meer, C. Sun, J. H. Snoeijer, and D. Lohse, Maximal Air Bubble Entrainment at Liquid-Drop Impact, Phys. Rev. Lett. 109, 264501 (2012).

[98] L. Xu, W. W. Zhang, and S. R. Nagel, Drop Splashing on a Dry Smooth Surface, Phys. Rev. Lett. 94, 184505 (2005).

[99] S. Thoroddsen, T. Etoh, K. Takehara, N. Ootsuka, and A. Hatsuki, The air bubble entrapped under a drop impacting on a solid surface, J. Fluid Mech. 545, 203 (2005).

[100] S. Thoroddsen, T. Etoh, and K. Takehara, High-speed imaging of drops and bubbles, Annu. Rev. Fluid Mech. 40, 257 (2008).

[101] S. Mandre, M. Mani, and M. P. Brenner, Precursors to Splashing of Liquid Droplets on a Solid Surface, Phys. Rev. Lett. 102, 134502 (2009).

[102] P. D. Hicks and R. Purvis, Air cushioning and bubble entrapment in three-dimensional droplet impacts, J. Fluid Mech. 649, 135 (2010).

[103] J. M. Kolinski, S. M. Rubinstein, S. Mandre, M. Mani, M. P. Brenner, D. A. Weitz, and L. Mahadevan, Skating On a Film of Air: Drops Impacting on a Surface, Phys. Rev. Lett. 108, 074503 (2012).

[104] C. Josserand and S. Thoroddsen, Drop impact on a solid surface, Annu. Rev. Fluid Mech. 48, 365 (2016).

[105] A. L. Yarin, I. V. Roisman, and C. Tropea, Collision Phenomena in Liquids and Solids (Cambridge University Press, Cambridge, 2017).

[106] R. C. A. van der Veen, T. Tran, D. Lohse, and C. Sun, Direct measurements of air layer profiles under impacting droplets using high-speed color interferometry, Phys. Rev. E 85, 026315 (2012). 
[107] R. Mettin, I. Akhatov, U. Parlitz, C. D. Ohl, and W. Lauterborn, Bjerknes forces between small cavitation bubbles in a strong acoustic field, Phys. Rev. E 56, 2924 (1997).

[108] N. Bremond, M. Arora, C. D. Ohl, and D. Lohse, Controlled Multi-Bubble Surface Cavitation, Phys. Rev. Lett. 96, 224501 (2006).

[109] N. Bremond, M. Arora, S. Dammer, and D. Lohse, Interaction of cavitation bubbles on a wall, Phys. Fluids 18, 121505 (2006).

[110] B. M. Borkent, S. Gekle, A. Prosperetti, and D. Lohse, Nucleation threshold and deactivation mechanisms of nanoscopic cavitation nuclei, Phys. Fluids 21, 102003 (2009).

[111] E. N. Harvey, D. K. Barnes, W. D. McElroy, A. H. Whiteley, D. C. Pease, and K. W. Cooper, Bubble formation in animals. I. Physical factors, J. Cell. Comp. Physiol. 24, 1 (1944).

[112] R. E. Apfel, The role of impurities in cavitation threshold determination, J. Acoust. Soc. Am. 48, 1179 (1970).

[113] L. A. Crum, Surface oscillations and jet development in pulsating bubbles, J. Phys. Colloques 40(C8), 285 (1979).

[114] A. A. Atchley and A. Prosperetti, The crevice model of bubble nucleation, J. Acoust. Soc. Am. 86, 1065 (1989).

[115] D. Rivas Fernandez, A. Prosperetti, A. G. Z. Aaldert, D. Lohse, and H. J. G. E. Gardeniers, Efficient sonochemistry through microbubbles generated with micromachined surfaces, Angew. Chem. Int. Ed. 49, 9699 (2010).

[116] D. Rivas Fernandez, B. Verhaagen, J. R. T. Seddon, A. G. Zijlstra, L. M. Jiang, L. W. M. van der Sluis, M. Versluis, D. Lohse, and H. J. G. E. Gardeniers, Localized removal of layers of metal, polymer, or biomaterial by ultrasound cavitation bubbles, Biomicrofluidics 6, 034114 (2012).

[117] D. N. Guzman, Y. Xie, S. Chen, D. Rivas Fernandez, C. Sun, D. Lohse, and G. Ahlers, Heat-flux enhancement by vapour-bubble nucleation in Rayleigh-Bénard turbulence, J. Fluid Mech. 787, 331 (2016).

[118] S.-T. Lou, Z.-Q. Ouyang, Y. Zhang, X.-J. Li, J. Hu, M.-Q. Li, and F.-J. Yang, Nanobubbles on solid surface imaged by atomic force microscopy, J. Vac. Sci. Technol. B 18, 2573 (2000).

[119] N. Ishida, T. Inoue, M. Miyahara, and K. Higashitani, Nano bubbles on a hydrophobic surface in water observed by tapping-mode atomic force microscopy, Langmuir 16, 6377 (2000).

[120] J. W. G. Tyrrell and P. Attard, Atomic force microscope images of nanbubbles on a hydrophobic surface and corresponding force-separation data, Langmuir 18, 160 (2002).

[121] D. Lohse and X. Zhang, Surface nanobubble and surface nanodroplets, Rev. Mod. Phys. 87, 981 (2015).

[122] X. H. Zhang, X. D. Zhang, S. T. Lou, Z. X. Zhang, J. L. Sun, and J. Hu, Degassing and temperature effects on the formation of nanobubbles at the mica/water interface, Langmuir 20, 3813 (2004).

[123] X. Zhang and W. Ducker, Formation of interfacial nanodroplets through changes in solvent quality, Langmuir 23, 12478 (2007).

[124] W. A. Ducker, Contact angle and stability of interfacial nanobubbles, Langmuir 25, 8907 (2009).

[125] N. Hain, D. Wesner, S. I. Druzhinin, and H. Schönherr, Surface nanobubbles studied by time-resolved fluorescence microscopy methods combined with AFM: The impact of surface treatment on nanobubble nucleation, Langmuir 32, 11155 (2015).

[126] B. Song, W. Walczyk, and H. Schönherr, Contact angles of surface nanobubbles on mixed self-assembled monolayers with systematically varied macroscopic wettability by atomic force microscopy, Langmuir 27, 8223 (2011).

[127] W. Walczyk and H. Schönherr, Dimensions and the profile of surface nanobubbles: Tip-nanobubble interactions and nanobubble deformation in atomic force microscopy, Langmuir 30, 11955 (2014).

[128] J. H. Weijs, J. H. Snoeijer, and D. Lohse, Surface Nanobubbles: Formation and Universality of the Contact Angle, Phys. Rev. Lett. 108, 104501 (2012).

[129] X. Zhang, D. Y. C. Chan, D. Wang, and N. Maeda, Stability of interfacial nanobubbles, Langmuir 29, 1017 (2013).

[130] R. G. Picknett and R. Bexon, The evaporation of sessile or pendant drops in still air, J. Colloid Interface Sci. 61, 336 (1977).

[131] A. M. Cazabat and G. Guéna, Evaporation of macroscopic sessile droplets, Soft Matter 6, 2591 (2010). 
[132] J. F. Joanny and P. G. de Gennes, A model for contact angle hysteresis, J. Chem. Phys. 81, 552 (1984).

[133] P. G. de Gennes, Wetting: Statics and dynamics, Rev. Mod. Phys. 57, 827 (1985).

[134] D. Lohse and X. Zhang, Pinning and gas oversaturation imply stable single surface nanobubble, Phys. Rev. E 91, 031003(R) (2015).

[135] Y. O. Popov, Evaporative deposition patterns: Spatial dimensions of the deposit, Phys. Rev. E 71, 036313 (2005).

[136] R. D. Deegan, O. Bakajin, T. F. Dupont, G. Huber, S. R. Nagel, and T. A. Witten, Capillary flow as the cause of ring stains from dried liquid drops, Nature (London) 389, 827 (1997).

[137] A. G. Marin, H. Gelderblom, D. Lohse, and J. H. Snoeijer, Order-to-Disorder Transition in Ring-Shaped Colloidal Stains, Phys. Rev. Lett. 107, 085502 (2011).

[138] X. Zhu, R. Verzicco, X. Zhang, and D. Lohse, Diffusive interaction of multiple surface nanobubbles: Shrinkage, growth, and coarsening, Soft Matter 14, 2006 (2018).

[139] S. Maheshwari, M. van der Hoef, X. Zhang, and D. Lohse, Stability of surface nanobubbles: A molecular dynamics study, Langmuir 32, 11116 (2016).

[140] J. H. Weijs and D. Lohse, Why Surface Nanobubbles Live for Hours, Phys. Rev. Lett. 110, 054501 (2013).

[141] S. Peng, T. L. Mega, and X. Zhang, Collective effects in microbubble growth by solvent exchange, Langmuir 32, 11265 (2016).

[142] S. Peng, V. Spandan, R. Verzicco, D. Lohse, and X. H. Zhang, Growth dynamics of microbubbles on microcavity arrays by solvent exchange, J. Colloid Interface Sci. 532, 103 (2018).

[143] X. H. Zhang, J. M. Ren, H. J. Yang, Y. H. He, J. F. Tan, and G. G. Qiao, From transient nanodroplets to permanent nanolenses, Soft Matter 8, 4314 (2012).

[144] X. Zhang, J. Wang, L. Bao, E. Dietrich, R. C. A. van der Veen, S. Peng, J. Friend, H. J. W. Zandvliet, L. Yeo, and D. Lohse, Mixed mode of dissolving immersed microdroplets at a solid-water interface, Soft Matter 11, 1889 (2015).

[145] P. W. Voorhees, The theory of Ostwald ripening, J. Stat. Phys. 38, 231 (1985).

[146] B. Dollet and D. Lohse, Pinning stabilizes neighboring surface nanobubbles against Ostwald ripening, Langmuir 32, 11335 (2016).

[147] S. Maheshwari, M. van der Hoef, J. Rodríguez Rodríguez, and D. Lohse, Leakiness of pinned neighboring surface nanobubbles induced by strong gas-surface interaction, ACS Nano 12, 2603 (2018).

[148] X. Zhang, Z. Lu, H. Tan, L. Bao, Y. He, C. Sun, and D. Lohse, Formation of surface nanodroplets under controlled flow conditions, Proc. Nat. Acad. Sci. USA 112, 9253 (2015).

[149] L. Bao, Z. Werbiuk, D. Lohse, and Z. Zhang, Controlling the growth modes of femtoliter sessile droplets nucleating on chemically patterned surfaces, J. Phys. Chem. Lett. 7, 1055 (2016).

[150] L. Bao, V. Spandan, Y. Yang, B. Dyett, R. Verzicco, D. Lohse, and X. Zhang, Flow-induced dissolution of femtoliter surface droplet arrays, Lab Chip 18, 1066 (2018).

[151] F. Pregl, Die Quantitative Organische Mikroanalyse (Springer, Berlin, 1917).

[152] A. Jain and K. K. Verma, Recent advances in applications of single-drop microextraction: A review, Anal. Chim. Acta 706, 37 (2011).

[153] M. Rezaee, Y. Assadi, M. R. M. Hosseinia, E. Aghaee, F. Ahmadi, and S. Berijani, Determination of organic compounds in water using dispersive liquid-liquid microextraction, J. Chromatogr. A 1116, 1 (2006).

[154] M. Rezaee, Y. Yamini, and M. Faraji, Evolution of dispersive liquid-liquid microextraction method, J. Chromatogr. A 1217, 2342 (2010).

[155] A. Zgola-Grzeskowiak and T. Grzeskowiak, Dispersive liquid-liquid microextraction, Trends Anal. Chem. 30, 1382 (2011).

[156] D. Lohse, Towards controlled liquid-liquid microextraction, J. Fluid Mech. 804, 1 (2016).

[157] G. Wagner, P. Schlanstein, S. Fiehe, T. Kaufmann, R. Kopp, R. Bensberg, T. Schmitz-Rode, U. Steinseifer, and J. Arens, A novel approach in extracorporeal circulation: individual, integrated, and interactive heart-lung assist ( $\mathrm{I}^{3}$-Assist), Biomed. Eng.-Bio. Tech. 59, 125 (2013).

[158] S. Yang, P. Tsai, E. S. Kooij, A. Prosperetti, H. J. W. Zandvliet, and D. Lohse, Electrolytically generated nanobubbles on highly orientated pyrolytic graphite surfaces, Langmuir 25, 1466 (2009); Correction 
to Electrolytically generated nanobubbles on highly orientated pyrolytic graphite surfaces, 29, 5937 (2013).

[159] P. Lv, H. Le The, J. Eijkel, A. Van den Berg, X. Zhang, and D. Lohse, Growth and detachment of oxygen bubbles induced by gold-catalyzed decomposition of hydrogen peroxide, J. Phys. Chem. C 121, 20769 (2017).

[160] P. van der Linde, A. M. Soto, P. Peñas-López, J. Rodríguez-Rodríguez, D. Lohse, H. Gardeniers, D. van der Meer, and D. Fernández Rivas, Electrolysis-driven and pressure-controlled diffusive growth of successive bubbles on microstructured surfaces, Langmuir 33, 12873 (2017).

[161] A. Moreno Soto, S. R. German, H. Ren, D. van der Meer, D. Lohse, M. R. Edwards, and H. S. White, The nucleation rate of single $\mathrm{O}_{2}$ nanobubbles at Pt nanoelectrodes, Langmuir 34, 7309 (2018).

[162] O. R. Enriquez, C. Sun, D. Lohse, A. Prosperetti, and D. van der Meer, The quasi-static growth of $\mathrm{CO}_{2}$ bubbles, J. Fluid Mech. 741, R1 (2014).

[163] A. Moreno Soto, A. Prosperetti, D. Lohse, and D. van der Meer, Gas depletion through single gas bubble diffusive growth and its effect on subsequent bubbles, J. Fluid Mech. 831, 474 (2017).

[164] W. Fritz, Berechung des Maximalvolumens von Dampfblasen, Phys. Z. 36, 379 (1935).

[165] A. Moreno Soto, T. Maddalena, A. Fraters, D. van der Meer, and D. Lohse, Coalescence of diffusively growing gas bubbles, J. Fluid Mech. 846, 143 (2018).

[166] J. Magnaudet and I. Eames, The motion of high-Reynolds number bubbles in inhomogeneous flows, Annu. Rev. Fluid Mech. 32, 659 (2000).

[167] J. M. Rensen, D. Bosman, J. Magnaudet, C. D. Ohl, A. Prosperetti, R. Tögel, M. Versluis, and D. Lohse, Spiraling Bubbles: How Acoustic and Hydrodynamic Forces Compete, Phys. Rev. Lett. 86, 4819 (2001).

[168] D. Lohse and A. Prosperetti, Controlling bubbles, J. Phys.: Condens. Matter 15, S415 (2003).

[169] E. A. van Nierop, J. J. Bluemink, S. Luther, J. Magnaudet, A. Prosperetti, and D. Lohse, Drag and lift forces on bubbles in a rotating flow, J. Fluid Mech. 571, 439 (2007).

[170] J. Magnaudet and D. Legendre, The viscous drag force on a spherical bubble with a time-dependent radius, Phys. Fluids 10, 550 (1998).

[171] R. Toegel, S. Luther, and D. Lohse, Viscosity Destabilizes Sonoluminescing Bubbles, Phys. Rev. Lett. 96, 114301 (2006).

[172] Y. T. Didenko, W. B. McNamara, and K. S. Suslick, Molecular emission from single-bubble sonoluminescence, Nature (London) 407, 877 (2000).

[173] I. M. Mazzitelli, D. Lohse, and F. Toschi, The effect of microbubbles on developed turbulence, Phys. Fluids 15, L5 (2003).

[174] I. M. Mazzitelli, D. Lohse, and F. Toschi, On the relevance of the lift force in bubbly turbulence, J. Fluid Mech. 488, 283 (2003).

[175] M. R. Maxey and J. J. Riley, Equation of motion for a small rigid sphere in a nonuniform flow, Phys. Fluids 26, 883 (1983).

[176] C. T. Crowe, T. R. Troutt, and J. N. Chung, Numerical models for two-phase turbulent flows, Annu. Rev. Fluid Mech. 28, 11 (1996).

[177] S. Balachandar and J. K. Eaton, Turbulent dispersed multiphase flow, Annu. Rev. Fluid Mech. 42, 111 (2010).

[178] F. Toschi and E. Bodenschatz, Lagrangian properties of particles in turbulence, Annu. Rev. Fluid Mech. 41, 375 (2009).

[179] N. H. Thomas, T. R. Auton, K. J. Sene, and J. C. R. Hunt, in Gas Transfer at Water Surfaces, edited by W. Brutsaert and G. H. Jurka (Reidel, Berlin, 1984), p. 255.

[180] T. R. Auton, The lift force on a spherical body in a rotational flow, J. Fluid Mech. 183, 199 (1987).

[181] L. P. Wang and M. R. Maxey, The motion of microbubbles in a forced isotropic and homogeneous turbulence, Appl. Sci. Res. 51, 291 (1993).

[182] M. R. Maxey, E. J. Chang, and L. P. Wang, Simulation of interactions between microbubbles and turbulent flows, Appl. Mech. Rev. 46, S70 (1994).

[183] J. Magnaudet, M. Rivero, and J. Fabre, Accelerated flows past a rigid sphere or a spherical bubble, J. Fluid Mech. 284, 97 (1995). 
[184] G. Sridhar and J. Katz, Drag and lift forces on microscopic bubbles entrained by a vortex, Phys. Fluids 7, 389 (1995).

[185] E. Climent, Dispersion de bulles et modification du mouvement de la phase porteuse dans des ecoulements tourbillonnaires, Ph.D. thesis, Institut National Polytechnique Toulouse, 1996.

[186] E. Climent and J. Magnaudet, Large-Scale Simulations of Bubble-Induced Convection in a Liquid Layer, Phys. Rev. Lett. 82, 4827 (1999).

[187] P. D. M. Spelt and A. Biesheuvel, On the motion of gas bubbles in homogeneous isotropic flow, J. Fluid Mech. 336, 221 (1997).

[188] D. Legendre and J. Magnaudet, The lift force on a spherical bubble in a viscous linear shear flow, J. Fluid Mech. 368, 81 (1998).

[189] G. Sridhar and J. Katz, Effect of entrained bubbles on the structure of vortex rings, J. Fluid Mech. 397, 171 (1999).

[190] I. M. Mazzitelli and D. Lohse, Lagrangian statistics for fluid particles and bubbles in turbulence, New J. Phys. 6, 203 (2004).

[191] E. Calzavarini, M. Cencini, D. Lohse, and F. Toschi, Quantifying Turbulence Induced Segregation of Inertial Particles, Phys. Rev. Lett. 101, 084504 (2008).

[192] E. Calzavarini, M. Kerscher, D. Lohse, and F. Toschi, Dimensionality and morphology of particle and bubble clusters in turbulent flow, J. Fluid Mech. 607, 13 (2008).

[193] P. Oresta, R. Verzicco, D. Lohse, and A. Prosperetti, Heat transfer mechanisms in bubbly RayleighBénard convection, Phys. Rev. E 80, 026304 (2009).

[194] R. Lakkaraju, L. E. Schmidt, P. Oresta, F. Toschi, R. Verzicco, D. Lohse, and A. Prosperetti, Effect of vapor bubbles on velocity fluctuations and dissipation rates in bubbly Rayleigh-Bénard convection, Phys. Rev. E 84, 036312 (2011).

[195] R. Lakkaraju, R. J. A. M. Stevens, P. Oresta, R. Verzicco, D. Lohse, and A. Prosperetti, Heat transport in bubbling turbulent convection, Proc. Nat. Acad. Sci. USA 110, 9237 (2013).

[196] N. K. Madavan, S. Deutsch, and C. L. Merkle, Reduction of turbulent skin friction by micro-bubbles, Phys. Fluids 27, 356 (1984).

[197] N. K. Madavan, S. Deutsch, and C. L. Merkle, Measurements of local skin friction in a microbubblemodified turbulent boundary layer, J. Fluid Mech. 156, 237 (1985).

[198] Y. Kodama, A. Kakugawa, T. Takahashi, and H. Kawashima, Experimental studies on microbubbles and their applicability to ships for skin friction reduction, Int. J. Heat Fluid Flow 21, 582 (2000).

[199] D. P. M. van Gils, D. Narezo Guzman, C. Sun, and D. Lohse, The importance of bubble deformability for strong drag reduction in bubbly turbulent Taylor-Couette flow, J. Fluid Mech. 722, 317 (2013).

[200] S. L. Ceccio, Friction drag reduction of external flows with bubble and gas injection, Annu. Rev. Fluid Mech. 42, 183 (2010).

[201] Y. Murai, Frictional drag reduction by bubble injection, Exp. Fluids 55, 1773 (2014).

[202] I. Kumagai, Y. Takahashi, and Y. Murai, Power-saving device for air bubble generation using a hydrofoil to reduce ship drag: Theory, experiments, and application to ships, Ocean Eng. 95, 183 (2015).

[203] M. A. Fardin, C. Perge, and N. Taberlet, The hydrogen atom of fluid dynamics-Introduction to the Taylor-Couette flow for soft matter scientists, Soft Matter 10, 3523 (2014).

[204] S. Grossmann, D. Lohse, and C. Sun, High Reynolds number Taylor-Couette turbulence, Annu. Rev. Fluid Mech 48, 53 (2016).

[205] T. H. van den Berg, S. Luther, D. P. Lathrop, and D. Lohse, Drag Reduction in Bubbly Taylor-Couette Turbulence, Phys. Rev. Lett. 94, 044501 (2005).

[206] T. H. van den Berg, D. P. M. van Gils, D. P. Lathrop, and D. Lohse, Bubbly Turbulent Drag Reduction is a Boundary Layer Effect, Phys. Rev. Lett. 98, 084501 (2007).

[207] J. C. Lu, A. Fernandez, and G. Tryggvason, Drag reduction in a turbulent channel due to bubble injection, Phys. Fluids 17, 095102 (2005).

[208] S. Takagi, T. Ogasawara, and Y. Matsumoto, The effects of surfactant on the multiscale structure of bubbly flows, Philos. Trans. R. Soc. A 366, 2117 (2008).

[209] S. Takagi and Y. Matsumoto, Surfactant effects on bubble motion and bubbly flows, Annu. Rev. Fluid Mech. 43, 615 (2011). 
[210] D. P. M. van Gils, G. W. Bruggert, D. P. Lathrop, C. Sun, and D. Lohse, The Twente turbulent TaylorCouette $\left(\mathrm{T}^{3} \mathrm{C}\right)$ facility: Strongly turbulent (multi-phase) flow between independently rotating cylinders, Rev. Sci. Instrum. 82, 025105 (2011).

[211] R. A. Verschoof, R. C. A. van der Veen, C. Sun, and D. Lohse, Bubble Drag Reduction Requires Large Bubbles, Phys. Rev. Lett. 117, 104502 (2016).

[212] J. Lu and G. Tryggvason, Effect of bubble deformability in turbulent bubbly upflow in a vertical channel, Phys. Fluids 20, 040701 (2008).

[213] S. Dabiri, J. Lu, and G. Tryggvason, Transition between regimes of a vertical channel bubbly upflow due to bubble deformability, Phys. Fluids 25, 102110 (2013).

[214] Y. Murai, H. Oiwa, and Y. Takeda, Bubble behavior in a vertical Taylor-Couette flow, J. Phys.: Conf. Ser. 14, 143 (2005).

[215] K. Sugiyama, E. Calzavarini, and D. Lohse, Microbubble drag reduction in Taylor-Couette flow in the wavy vortex regime, J. Fluid Mech. 608, 21 (2008).

[216] V. Spandan, D. Lohse, and R. Verzicco, Drag reduction in numerical two-phase Taylor-Couette turbulence using an Euler-Lagrange approach, J. Fluid Mech. 798, 411 (2016).

[217] V. Spandan, R. Verzicco, and D. Lohse, Deformable ellipsoidal bubbles in Taylor-Couette flow with enhanced Euler-Lagrangian tracking, Phys. Rev. Fluids 2, 104304 (2017).

[218] A. Chouippe, E. Climent, D. Legendre, and C. Gabillet, Numerical simulation of bubble dispersion in turbulent Taylor-Couette flow, Phys. Fluids 26, 043304 (2014).

[219] G. N. Fokoua, C. Gabillet, A. Aubert, and C. Colin, Effect of bubble's arrangement on the viscous torque in bubbly Taylor-Couette flow, Phys. Fluids 27, 034105 (2015).

[220] P. Maffettone and M. Minale, Equation of change for ellipsoidal drops in viscous flow, J. Non-Newtonian Fluid Mech. 78, 227 (1998).

[221] L. Biferale, C. Meneveau, and R. Verzicco, Deformation statistics of sub-Kolmogorov-scale ellipsoidal neutrally buoyant drops in isotropic turbulence, J. Fluid Mech. 754, 184 (2014).

[222] V. Spandan, R. Verzicco, and D. Lohse, Physical mechanisms governing drag reduction in turbulent Taylor-Couette flow with finite-size deformable bubbles, J. Fluid Mech. 849, R3 (2018).

[223] R. A. Verschoof, D. Bakhuis, P. A. Bullee, S. G. Huisman, C. Sun, and D. Lohse, Air cavities at the inner cylinder of turbulent Taylor-Couette flow, Int. J. Multiphase Flow 105, 264 (2018).

[224] M. Versluis, High-speed imaging in fluids, Exp. Fluids 54, 1458 (2013).

[225] A. Prosperetti, Bubbles, Phys. Fluids 16, 1852 (2004). 